\title{
The Role of Occupants in Buildings' Energy Performance Gap: Myth or Reality?
}

\author{
Ardeshir Mahdavi ${ }^{1}$, Christiane Berger ${ }^{1, *}$, Hadeer Amin ${ }^{1}$, Eleni Ampatzi ${ }^{2}$, Rune Korsholm Andersen ${ }^{3} \mathbb{D}$, \\ Elie Azar ${ }^{4}$ (D), Verena M. Barthelmes ${ }^{5}$, Matteo Favero ${ }^{6}$, Jakob Hahn ${ }^{7}$ (D), Dolaana Khovalyg ${ }^{5}$, \\ Henrik N. Knudsen ${ }^{8}{ }^{(D}$, Alessandra Luna-Navarro ${ }^{9}$, Astrid Roetzel ${ }^{10}$, Fisayo C. Sangogboye ${ }^{11}$, \\ Marcel Schweiker ${ }^{12}$, Mahnameh Taheri ${ }^{13}\left(\mathbb{D}\right.$, Despoina Teli ${ }^{14}(\mathbb{D})$, Marianne Touchie ${ }^{15}(\mathbb{D}$ \\ and Silke Verbruggen 16 (D)
}

\section{check for} updates

Citation: Mahdavi, A.; Berger, C.; Amin, H.; Ampatzi, E.;

Andersen, R.K.; Azar, E.;

Barthelmes, V.M.; Favero, M.;

Hahn, J.; Khovalyg, D.; et al. The Role of Occupants in Buildings' Energy Performance Gap: Myth or Reality? Sustainability 2021, 13, 3146. https:// doi.org/10.3390/su13063146

Academic Editors: Antonio Caggiano and Francesco Leccese

Received: 22 January 2021

Accepted: 9 March 2021

Published: 12 March 2021

Publisher's Note: MDPI stays neutral with regard to jurisdictional claims in published maps and institutional affiliations.

Copyright: (c) 2021 by the authors. Licensee MDPI, Basel, Switzerland. This article is an open access article distributed under the terms and conditions of the Creative Commons Attribution (CC BY) license (https:/ / creativecommons.org/licenses/by/ $4.0 /)$
1 Department of Building Physics and Building Ecology, TU Wien, 1040 Vienna, Austria; amahdavi@tuwien.ac.at (A.M.); hadeer.amin@tuwien.ac.at (H.A.)

2 Welsh School of Architecture, Cardiff University, Bute Building, King Edward VII Ave., Cardiff CF10 3NB, UK; ampatzie@cf.ac.uk

3 Department of Civil Engineering, Technical University of Denmark, 2800 Kgs. Lyngby, Denmark; rva@byg.dtu.dk

4 Department of Industrial and Systems Engineering, Khalifa University of Science and Technology, Abu Dhabi 127788, United Arab Emirates; elie.azar@ku.ac.ae

5 Thermal Engineering for the Built Environment Laboratory (TEBEL), École Polytechnique Fédérale de Lausanne (EPFL), Passage du Cardinal 13B, 1700 Fribourg, Switzerland; verena.barthelmes@epfl.ch (V.M.B.); dolaana.khovalyg@epfl.ch (D.K.)

6 Department of Civil and Environmental Engineering, Norwegian University of Science and Technology, 7491 Trondheim, Norway; matteo.favero@ntnu.no

7 Research Institute CENERGIE-Center for Energy Efficient Buildings and Districts, Department 05: Building Services Engineering, Munich University of Applied Sciences, Lothstr. 34, 80335 Munich, Germany; jakob.hahn@hm.edu

8 Division of Sustainability, Energy and Indoor Environment, Department of the Built Environment, Aalborg University, A. C. Meyers Vænge 15, 2450 Copenhagen, Denmark; hnkn@build.aau.dk

9 Department of Engineering, University of Cambridge, Cambridge CB2 1PZ, UK; al786@cam.ac.uk

10 School of Architecture and Built Environment, Deakin University, Geelong, VIC 3220, Australia; astrid.roetzel@deakin.edu.au

11 Software Engineering Section, Mærsk Mckinney Møller Institute, University of Southern Denmark, 5230 Odense, Denmark; fsan@mmmi.sdu.dk

12 Institute for Occupational, Social and Environmental Medicine, Medical Faculty, RWTH Aachen University, 52074 Aachen, Germany; mschweiker@ukaachen.de

13 Arbnco Ltd., Glasgow G1 1RD, UK; mtaheri@arbnco.com

14 Division of Building Services Engineering, Department of Architecture and Civil Engineering, Chalmers University of Technology, SE-41296 Göteborg, Sweden; teli@chalmers.se

15 Department of Civil and Mineral Engineering, University of Toronto, Toronto, ON M5S 1A4, Canada; marianne.touchie@utoronto.ca

16 Research Group Building Physics, Department of Architecture and Urban Planning, Ghent University, 9000 Ghent, Belgium; silke.verbruggen@ugent.be

* Correspondence: christiane.berger@tuwien.ac.at; Tel.: +43-1-58801-27003

Abstract: Buildings' expected (projected, simulated) energy use frequently does not match actual observations. This is commonly referred to as the energy performance gap. As such, many factors can contribute to the disagreement between expectations and observations. These include, for instance, uncertainty about buildings' geometry, construction, systems, and weather conditions. However, the role of occupants in the energy performance gap has recently attracted much attention. It has even been suggested that occupants are the main cause of the energy performance gap. This, in turn, has led to suggestions that better models of occupant behavior can reduce the energy performance gap. The present effort aims at the review and evaluation of the evidence for such claims. To this end, a systematic literature search was conducted and relevant publications were identified and reviewed in detail. The review entailed the categorization of the studies according to the scope and strength of the evidence for occupants' role in the energy performance gap. Moreover, deployed calculation and monitoring methods, normalization procedures, and reported causes and magnitudes of the energy 
performance gap were documented and evaluated. The results suggest that the role of occupants as significant or exclusive contributors to the energy performance gap is not sufficiently substantiated by evidence.

Keywords: buildings; performance gap; energy; occupant behavior

\section{Introduction}

\subsection{Objectives}

There is not a unique and all-encompassing definition of the term "energy performance gap" (EPG). Indeed, it has different connotations in different domains and contexts. It is thus necessary to clarify, at the outset, our understanding of this term. First, the domain we focus on covers buildings. Second, the energy we refer to is what is required for the operation of buildings. This includes energy needed for space heating, cooling, lighting, ventilation, equipment, and appliances as well as domestic hot water (DHW). Third, the gap we talk about is the one between expected (i.e., estimated, calculated, computed, predicted) and actual building-related energy use [1]. Fourth, whereas the deviation of buildings' actual energy use from the predicted magnitude may have different causes, we specifically focus on the potential role of building occupants with regard to the emergence and extent of the EPG.

As such, the present paper entails a review of recent publications deemed to be relevant to the initial objectives of our inquiry. These could be formulated in terms of a number of basic questions:

(i) What is the general frequency and scope of publications that address a buildingrelated EPG?

(ii) Do these publications entail a clear and widely shared understanding of the meaning of the EPG?

(iii) What fraction of these publications suggests that building occupants are responsible for a significant share of the EPG?

(iv) What kind and level of evidence is provided for the purported role of occupants in the EPG?

(v) Assuming there is evidence for the existence and relevance of an occupant-caused EPG, does the study of the literature entail suggestions as to how it could be reduced?

It is of critical importance to understand what the present contribution is not concerned with. We do not question the assertion that occupants' patterns of presence and behavior in buildings can, in principle, influence buildings' energy performance. Such a possibility is entirely plausible. Aside from their numbers and patterns of their presence in buildings, occupants can - in most buildings-manipulate the control parameters of environmental control systems for heating, cooling, ventilation, and lighting. Instances of such parameters include temperature set-points and schedules for heating and cooling systems. Similarly, occupants' operation of luminaires, windows, blinds, as well as electrical equipment and appliances can impact mass and energy transfer processes in buildings and hence their overall energy performance. Such scenarios of occupants' impact on buildings' energy performance can be demonstrated via rational analyses and simulation studies [1,2]. However, there is a fundamental distinction to be made between the plausibility of various effects and phenomena on the one hand and the existence, extent, and frequency of their actual occurrence on the other hand. Whereas the former may be accepted merely on logical grounds, the latter requires empirical evidence. Consequently, in this paper we are predominantly concerned with the existence and quality of the evidence for the claim that occupants' carry the bulk of responsibility for building-related EPGs.

Note that the present paper considers existing publications in this area and does not include any direct statistical treatment of empirical data. Nonetheless, its underlying 
main objective may be formulated in terms of a qualitatively expressed null-hypothesis as follows:

There is no conclusive and sufficient evidence available for the claim that occupants' behavior is responsible for the bulk of building-related EPGs.

As such, the outcome of this review is expected to support the effort to find out if this null-hypothesis can be rejected.

\subsection{Motivation}

The scientific literature and case studies report the existence of a gap between the predicted and actual energy use of buildings. Instances of such a gap have been reported in relation to existing buildings, building retrofit projects, and new constructions. For retrofit projects, this so-called performance gap is split into a prebound and a rebound effect, while for new constructions no such distinction is made. The prebound effect describes the difference between the predicted and actual energy use before the renovation measures and the rebound effect denotes such difference after the completion of the project.

Building occupants and their preferences, needs, socioeconomic conditions, and interactions with the building are often held responsible for a large part of this EPG and the variation in energy use between nominally identical buildings. Whether accompanied by numbers or not, the alleged contribution of occupants to this gap is then used as an argument for the detailed study of occupant behavior (OB) and the introduction of ever more complex OB models for energy use prediction. Computing power and more advanced simulation tools are suggested to improve the accuracy of energy use predictions. If occupants are indeed a major contributor to the gap, then the incorporation of more accurate occupant models in the simulation models could alleviate the problem. However, before making the occupant a major culprit, the basis and evidence for the above claims need to be examined.

The motivation behind the present review is to ascertain if there is indeed sufficient evidence for the claim that OB is a major contributor to the EPG. This review is also expected to shed light on further questions. For instance, even if occupants could be shown to be responsible for a considerable fraction of the performance gap, to which extent could we enhance the reliability and predictive accuracy of OB models? More generally, would closing the EPG improve the process of designing more energy efficient buildings? The present contribution is also intended to contribute to the identification of shortcomings in research related to the EPG.

\subsection{Overview of the Paper}

Section 2 provides an overview of the study's approach, including the paper selection process, the key research directions explored, and how the data are synthesized to extract the relevant information. Section 3 presents the results of the review. The section starts with the descriptive statistics of the selected publications, followed by the characteristics of the buildings and occupants studied, the type of data used, and the normalization approaches applied to the data. The section then continues with a critical analysis of the reported magnitudes and causes of the EPG. Section 4 discusses the main findings, and puts those in the context of the objectives of the review, their implications, and their practical applications. Section 5 concludes with a high-level summary of the work and way forward.

\section{Approach}

\subsection{Selection Process and Key Review Aspects}

The literature search process aimed to collect papers that directly address and document the role of occupants as the cause of the EPG. The initial process included screening of the authors' individual repositories for relevant papers and unstructured literature searches using various databases. Next, a structured search process was followed using both the Scopus [3] and the Web of Science databases [4]. The strings used for the literature search are reported in Appendix A. This process included two steps: 
1. A first search that looked for the relevant terms (e.g., performance gap, rebound, prebound, gap) in either the title or the keywords;

2. A second search within these findings that looked for entries with variations of the terms "buildings" and "occupants" or "uncertainty".

The literature search was then further refined (using available filtering options in the two databases) in order to only include records:

(a) Published in English.

(b) In relevant "subject areas" (Scopus) or "categories" (Web of Science), and

(c) In relevant "source titles" (both databases).

The latter (c) was performed via refining by "source titles", whilst attention was paid so that relevant interdisciplinary studies were not mistakenly omitted.

This process, as illustrated in the Prisma diagram in Figure 1, identified 242 potentially relevant publications. A first screening step was performed considering titles and abstracts, reducing the list to 102 publications that were fully screened. This structured process identified 74 relevant publications that were not included in the initial compilation of known research (items included in authors' collections and identified via unstructured search). In the next step, all references cited by the identified articles were screened for relevance. The entire process identified 144 articles.

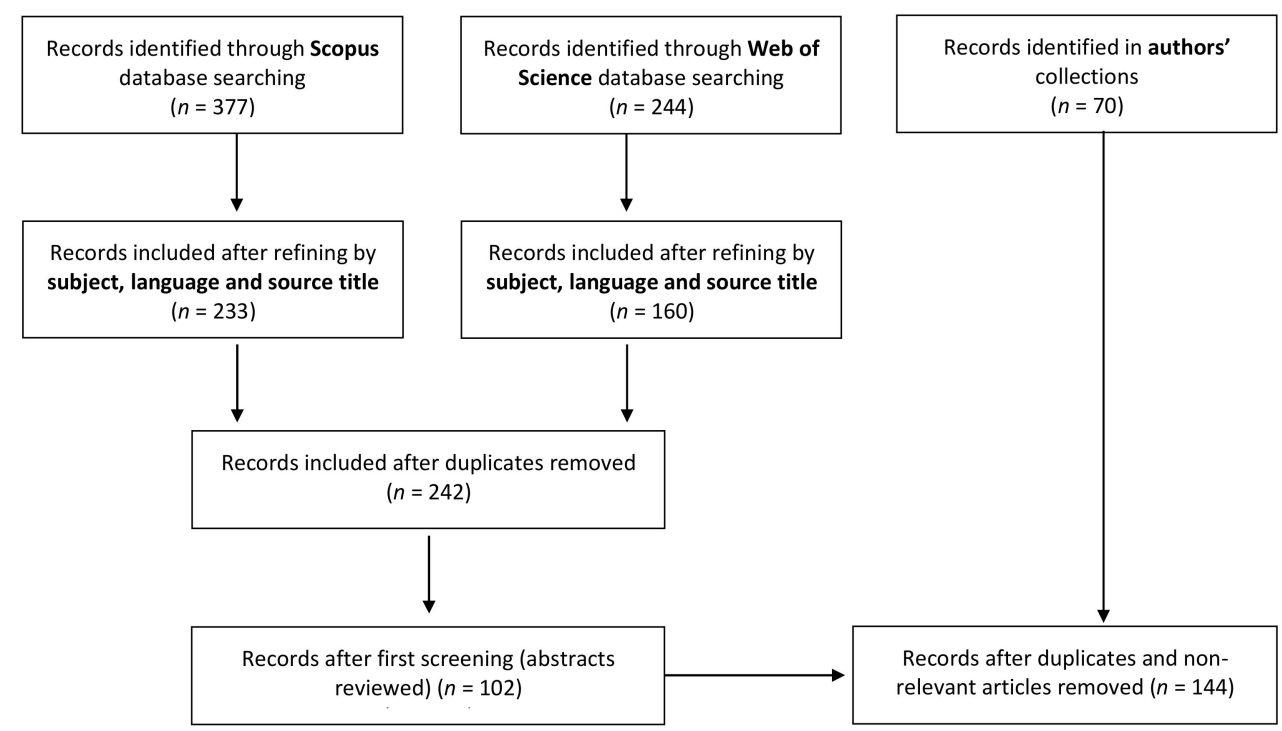

Figure 1. Process of identifying relevant publications.

Subsequently, the articles were split into two groups, i.e., those which directly addressed and documented the role of occupants as the cause of the performance gap ("main category") and those which addressed the performance gap without the strict requirement to provide evidence for the role of the occupants ("secondary category").

Lastly, a further high-level differentiation concerned the level of the entailed evidence for the EPG. Accordingly, the articles were divided into three groups: (i) the "gold" level denotes articles that contain empirical data of both energy use and occupant behavior, (ii) the "silver" level denotes articles that include empirical data only on energy use, and (iii) the "bronze" level denotes articles that may have included some occupant-related data but include no energy use data.

Table 1 provides key information for a subset of the articles with the above mentioned "gold label". References with the "silver" and "bronze" labels are listed in Appendix B, which includes a table with all reviewed publications. It includes, for each paper, summary information with regard to buildings, predicted energy, the source of occupant-related model assumptions, measured energy use, normalized energy data, and the magnitude of the EPG, together with primary conclusions. 
Table 1. Summary information regarding papers classified as "gold label".

\begin{tabular}{|c|c|c|c|c|c|c|c|c|c|c|}
\hline \multirow[b]{2}{*}{ Ref. } & \multirow[b]{2}{*}{$\begin{array}{l}\text { Building Type, } \\
\text { Location, } \\
\text { Construction } \\
\text { Date }\end{array}$} & \multirow[b]{2}{*}{$\begin{array}{c}\text { Predicted } \\
\text { Energy } \\
\text { Variable } \\
\text { (Method of } \\
\text { Prediction) }\end{array}$} & \multirow[b]{2}{*}{$\begin{array}{l}\text { Source of } \\
\text { Occupant- } \\
\text { Related } \\
\text { Model } \\
\text { Assumptions }\end{array}$} & \multicolumn{5}{|c|}{ Observations/Measurements } & \multirow[b]{2}{*}{$\begin{array}{c}\text { Normalized } \\
\text { Energy Data } \\
\text { (Normalization } \\
\text { Method) }\end{array}$} & \multirow[b]{2}{*}{$\underset{\text { Magnitude }}{\text { (in \%) }}$} \\
\hline & & & & $\begin{array}{l}\text { Duration } \\
\text { (Days) }\end{array}$ & $\begin{array}{l}\text { Measured } \\
\text { Energy Type } \\
\text { (Source of } \\
\text { Energy Data) }\end{array}$ & $\begin{array}{l}\text { Indoor } \\
\text { Conditions } \\
\text { (Source of } \\
\text { Data) }\end{array}$ & $\begin{array}{c}\text { Outdoor } \\
\text { Conditions } \\
\text { (Source of } \\
\text { Data) }\end{array}$ & $\begin{array}{c}\text { Observed } \\
\text { Occupant } \\
\text { Behavior } \\
\text { (Source of User } \\
\text { Behavior Data) }\end{array}$ & & \\
\hline [5] & $\begin{array}{c}\text { Residential; } \\
\text { Switzerland; } 2009\end{array}$ & $\begin{array}{c}\text { Heating load } \\
\text { (simulation) }\end{array}$ & $\begin{array}{c}\text { (Standard) } \\
\text { Swiss Society } \\
\text { of Engineers } \\
\text { and Architects } \\
\text { (SIA) }\end{array}$ & 730 & $\begin{array}{l}\text { Electricity, } \\
\text { pellet, solar } \\
\text { (energy } \\
\text { metering, } \\
\text { energy bills, in } \\
\text { situ measure- } \\
\text { ments) }\end{array}$ & $\begin{array}{c}\text { Air } \\
\text { temperature } \\
\text { (NA) }\end{array}$ & NA & $\begin{array}{c}\text { Occupancy, } \\
\text { window } \\
\text { opening } \\
\text { behavior, use of } \\
\text { shades } \\
\text { (observations) }\end{array}$ & $\begin{array}{l}\text { Energy } \\
\text { consumption for } \\
\text { heating and hot } \\
\text { water (adjusted } \\
\text { for degree days) }\end{array}$ & $\begin{array}{c}-1 \% \\
\text { (simulation), } \\
81 \% \text { (SIA } \\
\text { calculation) }\end{array}$ \\
\hline [6] & $\begin{array}{l}\text { Residential; } \\
\text { United Kingdom; } \\
2007\end{array}$ & $\begin{array}{l}\text { Heating load } \\
\text { (simulation, } \\
\text { standard } \\
\text { assessment } \\
\text { procedure } \\
\text { calculation) }\end{array}$ & $\begin{array}{l}\text { Standard } \\
\text { Assessment } \\
\text { Procedure } \\
\text { (SAP) } \\
\text { assumptions }\end{array}$ & 365 & $\begin{array}{l}\text { Electricity, } \\
\text { biomass, } \\
\text { district } \\
\text { heating } \\
\text { (energy } \\
\text { metering) }\end{array}$ & NA & NA & $\begin{array}{c}\text { Temperature } \\
\text { set-points, } \\
\text { window } \\
\text { behavior, } \\
\text { MVHR use, } \\
\text { programmer } \\
\text { control (survey) }\end{array}$ & NA & $\begin{array}{l}7 \% \text { higher on } \\
\text { average, for } \\
\text { the surveyed } \\
\text { sample it } \\
\text { ranged from } \\
-48 \% \text { to } 67 \%\end{array}$ \\
\hline [7] & $\begin{array}{l}\text { Residential; China; } \\
2007\end{array}$ & $\begin{array}{l}\text { Electricity } \\
\quad \text { (NA) }\end{array}$ & NA & 365 & $\begin{array}{l}\text { Electricity } \\
\text { (energy } \\
\text { metering) }\end{array}$ & $\begin{array}{c}\text { Air } \\
\text { temperature, } \\
\text { relative } \\
\text { humidity } \\
\text { (Testo 175-H2 } \\
\text { data loggers) }\end{array}$ & NA & $\begin{array}{l}\text { Length and } \\
\text { frequency of } \\
\text { daily use of } \\
\text { lighting and } \\
\text { appliances } \\
\text { (survey (paper } \\
\text { questionnaire)) }\end{array}$ & NA & NA \\
\hline [8] & $\begin{array}{l}\text { Non-residential; } \\
\text { England; } 2008\end{array}$ & $\begin{array}{c}\text { Energy } \\
\text { demand } \\
\text { (simulation) }\end{array}$ & $\begin{array}{l}\text { International } \\
\text { Performance } \\
\text { Measurement } \\
\text { and } \\
\text { Verification } \\
\text { Protocol } \\
\text { (IPMVP) }\end{array}$ & 730 & $\begin{array}{l}\text { Electricity, } \\
\text { natural gas } \\
\text { (energy) bills, } \\
\text { energy } \\
\text { metering) }\end{array}$ & NA & NA & $\begin{array}{l}\text { Occupancy, } \\
\text { temperature } \\
\text { set-points, } \\
\text { ventilation rates, } \\
\text { schedules of } \\
\text { operation for } \\
\text { heating, } \\
\text { ventilation } \\
\text { systems (POE } \\
\text { (interviews, } \\
\text { on-site } \\
\text { observations)) }\end{array}$ & $\begin{array}{c}\text { Gas } \\
\text { consumption for } \\
\text { heating, } \\
\text { electricity } \\
\text { consumption for } \\
\text { external lights } \\
\text { and lifts } \\
\text { (adjusted for } \\
\text { degree days, } \\
\text { NA) }\end{array}$ & $3.1 \%$ \\
\hline
\end{tabular}


Table 1. Cont.

\begin{tabular}{|c|c|c|c|c|c|c|c|c|c|c|}
\hline \multirow[b]{2}{*}{ Ref. } & \multirow[b]{2}{*}{$\begin{array}{c}\text { Building Type, } \\
\text { Location, } \\
\text { Construction } \\
\text { Date }\end{array}$} & \multirow[b]{2}{*}{$\begin{array}{l}\text { Predicted } \\
\text { Energy } \\
\text { Variable } \\
\text { (Method of } \\
\text { Prediction) }\end{array}$} & \multirow[b]{2}{*}{$\begin{array}{c}\text { Source of } \\
\text { Occupant- } \\
\text { Related } \\
\text { Model } \\
\text { Assumptions }\end{array}$} & \multicolumn{5}{|c|}{ Observations/Measurements } & \multirow[b]{2}{*}{$\begin{array}{c}\text { Normalized } \\
\text { Energy Data } \\
\text { (Normalization } \\
\text { Method) }\end{array}$} & \multirow[b]{2}{*}{$\underset{\substack{\text { Magnitude } \\
\text { (in \%) }}}{\text { EPG }}$} \\
\hline & & & & $\begin{array}{l}\text { Duration } \\
\text { (Days) }\end{array}$ & $\begin{array}{c}\text { Measured } \\
\text { Energy Type } \\
\text { (Source of } \\
\text { Energy Data) }\end{array}$ & $\begin{array}{l}\text { Indoor } \\
\text { Conditions } \\
\text { (Source of } \\
\text { Data) }\end{array}$ & $\begin{array}{c}\text { Outdoor } \\
\text { Conditions } \\
\text { (Source of } \\
\text { Data) }\end{array}$ & $\begin{array}{c}\text { Observed } \\
\text { Occupant } \\
\text { Behavior } \\
\text { (Source of User } \\
\text { Behavior Data) }\end{array}$ & & \\
\hline [9] & $\begin{array}{c}\text { Residential; } \\
\text { Netherlands; NA }\end{array}$ & NA & NA & 1095 & $\begin{array}{l}\text { Gas, electricity } \\
\text { (energy bills) }\end{array}$ & NA & $\begin{array}{c}\text { Weather } \\
\text { (national } \\
\text { climatic data) }\end{array}$ & $\begin{array}{l}\text { Occupancy, } \\
\text { temperature } \\
\text { set-point, } \\
\text { ventilation } \\
\text { behavior } \\
\text { (survey) } \\
\end{array}$ & $\begin{array}{c}\text { Energy } \\
\text { consumption for } \\
\text { heating and hot } \\
\text { water (NA) }\end{array}$ & NA \\
\hline$[10]$ & $\begin{array}{l}\text { Residential; } \\
\text { Belgium; 2008, } \\
1960\end{array}$ & $\begin{array}{l}\text { Heating load } \\
\text { (standard } \\
\text { (Belgian } \\
\text { EPBD)) }\end{array}$ & Standard & 2920 & $\begin{array}{l}\text { Gas (energy } \\
\text { bills) }\end{array}$ & $\begin{array}{c}\text { Air } \\
\text { temperature, } \\
\text { relative } \\
\text { humidity, } \mathrm{CO}_{2} \\
\text { (sensors) }\end{array}$ & $\begin{array}{c}\text { Weather } \\
\text { (national } \\
\text { climatic data) }\end{array}$ & $\begin{array}{l}\text { Occupancy, } \\
\text { thermostat use; } \\
\text { set-point and } \\
\text { frequency, } \\
\text { ventilation } \\
\text { behavior; } \\
\text { mechanical } \\
\text { system and } \\
\text { window } \\
\text { opening } \\
\text { (survey) }\end{array}$ & $\begin{array}{c}\text { Gas } \\
\text { consumption for } \\
\text { heating and hot } \\
\text { water (adjusted } \\
\text { for degree days) }\end{array}$ & $\begin{array}{l}\text { Case study } 1: \\
25 \% \text { to } 125 \% \text {, } \\
\text { case study 2: } \\
25 \% \text { to } 75 \%\end{array}$ \\
\hline [11] & $\begin{array}{l}\text { Residential; Italy; } \\
\text { NA }\end{array}$ & $\begin{array}{l}\text { Heating load } \\
\text { (energy } \\
\text { certificate) }\end{array}$ & Standard & 105 & $\begin{array}{l}\text { Ground water } \\
\text { supply heat, } \\
\text { electricity, } \\
\text { solar energy, } \\
\text { gas (energy } \\
\text { metering) }\end{array}$ & $\begin{array}{c}\text { Air } \\
\text { temperature } \\
\text { (data loggers) }\end{array}$ & NA & $\begin{array}{l}\text { Occupancy, } \\
\text { temperature } \\
\text { setting, use of } \\
\text { windows and } \\
\text { shutter (sensors) }\end{array}$ & $\begin{array}{c}\text { Energy } \\
\text { consumption for } \\
\text { heating and } \\
\text { DHW (adjusted } \\
\text { for degree days) }\end{array}$ & $\begin{array}{c}\text { Heating: } 77 \% \\
\text { to } 222 \% \text {, } \\
\text { DHW: } 96 \%\end{array}$ \\
\hline
\end{tabular}


Table 1. Cont.

\begin{tabular}{|c|c|c|c|c|c|c|c|c|c|c|}
\hline \multirow[b]{2}{*}{ Ref. } & \multirow[b]{2}{*}{$\begin{array}{c}\text { Building Type, } \\
\text { Location, } \\
\text { Construction } \\
\text { Date }\end{array}$} & \multirow[b]{2}{*}{$\begin{array}{l}\text { Predicted } \\
\text { Energy } \\
\text { Variable } \\
\text { (Method of } \\
\text { Prediction) }\end{array}$} & \multirow[b]{2}{*}{$\begin{array}{l}\text { Source of } \\
\text { Occupant- } \\
\text { Related } \\
\text { Model } \\
\text { Assumptions }\end{array}$} & \multicolumn{5}{|c|}{ Observations/Measurements } & \multirow[b]{2}{*}{$\begin{array}{c}\text { Normalized } \\
\text { Energy Data } \\
\text { (Normalization } \\
\text { Method) }\end{array}$} & \multirow[b]{2}{*}{$\underset{\substack{\text { EPG } \\
\text { (in \%) }}}{\text { EPnitude }}$} \\
\hline & & & & $\begin{array}{c}\text { Duration } \\
\text { (Days) }\end{array}$ & $\begin{array}{c}\text { Measured } \\
\text { Energy Type } \\
\text { (Source of } \\
\text { Energy Data) }\end{array}$ & $\begin{array}{l}\text { Indoor } \\
\text { Conditions } \\
\text { (Source of } \\
\text { Data) }\end{array}$ & $\begin{array}{l}\text { Outdoor } \\
\text { Conditions } \\
\text { (Source of } \\
\text { Data) }\end{array}$ & $\begin{array}{c}\text { Observed } \\
\text { Occupant } \\
\text { Behavior } \\
\text { (Source of User } \\
\text { Behavior Data) }\end{array}$ & & \\
\hline [12] & $\begin{array}{c}\text { Residential; } \\
\text { Austria; } \\
\text { 1968-1975 }\end{array}$ & $\begin{array}{l}\text { Heating load } \\
\text { (energy } \\
\text { certificate, } \\
\text { simulation) }\end{array}$ & Standard & 210 & $\begin{array}{l}\text { Electricity, gas, } \\
\text { oil, district } \\
\text { heating } \\
\text { (energy bills) }\end{array}$ & $\begin{array}{c}\text { Air } \\
\text { temperature, } \\
\text { relative } \\
\text { humidity } \\
\text { (sensors) }\end{array}$ & $\begin{array}{l}\text { Weather data } \\
\text { (local weather } \\
\text { stations) }\end{array}$ & $\begin{array}{c}\text { Window } \\
\text { opening } \\
\text { (interviews) }\end{array}$ & $\begin{array}{c}\text { Weather data, } \\
\text { indoor } \\
\text { temperature, air } \\
\text { change rate, } \\
\text { energy } \\
\text { consumption for } \\
\text { heating and hot } \\
\text { water (NA, take } \\
\text { monthly mean } \\
\text { indoor } \\
\text { temperatures, } \\
\text { consider air } \\
\text { change rate } \\
\text { higher than the } \\
\text { energy } \\
\text { certificate } \\
\text { assumptions by } \\
\text { a factor of 4-6) }\end{array}$ & $400 \%$ to $600 \%$ \\
\hline [13] & $\begin{array}{l}\text { Residential; Iran; } \\
\text { NA }\end{array}$ & $\begin{array}{c}\text { Energy } \\
\text { demand } \\
\text { (simulation) }\end{array}$ & Surveys & 1095 & $\begin{array}{l}\text { Gas, electricity } \\
\text { (energy bills, } \\
\text { field studies } \\
\text { and } \\
\text { interviews) }\end{array}$ & NA & NA & $\begin{array}{c}\text { Occupation } \\
\text { schedule, usage } \\
\text { of appliances, } \\
\text { lighting, heating } \\
\text { and cooling } \\
\text { appliances } \\
\text { (survey) }\end{array}$ & NA & $13.5 \%$ to $25 \%$ \\
\hline
\end{tabular}


Table 1. Cont.

\begin{tabular}{|c|c|c|c|c|c|c|c|c|c|c|}
\hline \multirow[b]{2}{*}{ Ref. } & \multirow[b]{2}{*}{$\begin{array}{l}\text { Building Type, } \\
\text { Location, } \\
\text { Construction } \\
\text { Date }\end{array}$} & \multirow[b]{2}{*}{$\begin{array}{l}\text { Predicted } \\
\text { Energy } \\
\text { Variable } \\
\text { (Method of } \\
\text { Prediction) }\end{array}$} & \multirow[b]{2}{*}{$\begin{array}{l}\text { Source of } \\
\text { Occupant- } \\
\text { Related } \\
\text { Model } \\
\text { Assumptions }\end{array}$} & \multicolumn{5}{|c|}{ Observations/Measurements } & \multirow[b]{2}{*}{$\begin{array}{c}\text { Normalized } \\
\text { Energy Data } \\
\text { (Normalization } \\
\text { Method) }\end{array}$} & \multirow[b]{2}{*}{$\underset{\substack{\text { Magnitude } \\
\text { (in \%) }}}{\text { EPG }}$} \\
\hline & & & & $\begin{array}{l}\text { Duration } \\
\text { (Days) }\end{array}$ & $\begin{array}{c}\text { Measured } \\
\text { Energy Type } \\
\text { (Source of } \\
\text { Energy Data) }\end{array}$ & $\begin{array}{l}\text { Indoor } \\
\text { Conditions } \\
\text { (Source of } \\
\text { Data) }\end{array}$ & $\begin{array}{c}\text { Outdoor } \\
\text { Conditions } \\
\text { (Source of } \\
\text { Data) }\end{array}$ & $\begin{array}{c}\text { Observed } \\
\text { Occupant } \\
\text { Behavior } \\
\text { (Source of User } \\
\text { Behavior Data) }\end{array}$ & & \\
\hline [14] & $\begin{array}{l}\text { Residential; UK; } \\
\text { 2011-2012 }\end{array}$ & $\begin{array}{c}\text { Energy } \\
\text { demand } \\
\text { (Standard } \\
\text { Assessment } \\
\text { Procedure } \\
(\text { SAP)) }\end{array}$ & $\begin{array}{l}\text { Standard } \\
\text { Assessment } \\
\text { Procedure } \\
\text { (SAP) }\end{array}$ & 365 & $\begin{array}{c}\text { Gas, PV, } \\
\text { electricity } \\
\text { (energy bills) }\end{array}$ & $\begin{array}{c}\text { Temperature, } \\
\text { relative } \\
\text { humidity (RH) } \\
\text { and } \mathrm{CO}_{2} \\
\text { (sensors) }\end{array}$ & NA & $\begin{array}{l}\text { Occupancy, (A) } \\
\text { opening and } \\
\text { closing of doors } \\
\text { and windows } \\
\text { (sensor), (B) } \\
\text { washing and } \\
\text { showering } \\
\text { regimes and } \\
\text { thermostat } \\
\text { settings } \\
\text { (self-completion } \\
\text { activity logging } \\
\text { of occupants) }\end{array}$ & NA & Up to $250 \%$ \\
\hline [15] & $\begin{array}{l}\text { Residential; } \\
\text { Landshut, } \\
\text { Germany; } 2011\end{array}$ & $\begin{array}{l}\text { Heating load } \\
\text { (Norm } \\
\text { DIN V 4108-6) }\end{array}$ & $\begin{array}{l}\text { Code } \\
\text { calculation, } \\
\text { simulations }\end{array}$ & 1460 & $\begin{array}{l}\text { Heating } \\
\text { (thermal } \\
\text { energy), DHW, } \\
\text { electricity } \\
\text { (sensors) }\end{array}$ & $\begin{array}{c}\text { Air } \\
\text { temperature, } \\
\mathrm{CO}_{2} \text {, humidity } \\
\text { (sensors) }\end{array}$ & $\begin{array}{c}\text { Air } \\
\text { temperature, } \\
\text { windspeed, } \\
\text { humidity, rain, } \\
\text { radiation } \\
\text { (weather } \\
\text { station on the } \\
\text { roof) }\end{array}$ & $\begin{array}{l}\text { Thermostats, } \\
\text { energy use } \\
\text { behavior } \\
\text { (monitoring, } \\
\text { meta data) }\end{array}$ & $\begin{array}{c}\text { Heating energy } \\
\text { (adjusted for } \\
\text { degree days), } \\
\text { square meter } \\
\text { normalized vs. } \\
\text { person } \\
\text { normalized- } \\
\text { DHW (cross } \\
\text { validation } \\
\text { between units) }\end{array}$ & $\begin{array}{c}\text { Heating: avg. } \\
30 \% \text {, } \\
\text { domestic hot } \\
\text { water: avg. } \\
-26 \%\end{array}$ \\
\hline [16] & $\begin{array}{c}\text { Residential; } \\
\text { Aarhus, Denmark; } \\
2017\end{array}$ & $\begin{array}{c}\text { Energy } \\
\text { demand } \\
\text { (simulation) }\end{array}$ & $\begin{array}{l}\text { Occupancy } \\
\text { schedules } \\
\text { (survey, } \\
\text { databases) }\end{array}$ & 180 & $\begin{array}{l}\text { Electricity, } \\
\text { district } \\
\text { heating } \\
\text { (energy } \\
\text { metering) }\end{array}$ & $\begin{array}{c}\text { Air } \\
\text { temperature, } \\
\text { air humidity, } \\
\text { ventilation } \\
\text { speed } \\
\mathrm{CO}_{2} \text { (sensors) }\end{array}$ & $\begin{array}{c}\text { Air } \\
\text { temperature } \\
\text { (sensors) }\end{array}$ & $\begin{array}{c}\text { Occupancy } \\
\left(\mathrm{CO}_{2} \text { sensor), }\right. \\
\text { appliance usage } \\
\text { (power meter } \\
\text { per appliance) }\end{array}$ & NA & $\begin{array}{c}\text { Heating: } \\
\text {-2.43\% } \\
\text { electricity: } \\
0.2 \%\end{array}$ \\
\hline
\end{tabular}


Table 1. Cont.

\begin{tabular}{|c|c|c|c|c|c|c|c|c|c|c|}
\hline \multirow[b]{2}{*}{ Ref. } & \multirow[b]{2}{*}{$\begin{array}{l}\text { Building Type, } \\
\text { Location, } \\
\text { Construction } \\
\text { Date }\end{array}$} & \multirow[b]{2}{*}{$\begin{array}{c}\text { Predicted } \\
\text { Energy } \\
\text { Variable } \\
\text { (Method of } \\
\text { Prediction) }\end{array}$} & \multirow[b]{2}{*}{$\begin{array}{c}\text { Source of } \\
\text { Occupant- } \\
\text { Related } \\
\text { Model } \\
\text { Assumptions }\end{array}$} & \multicolumn{5}{|c|}{ Observations/Measurements } & \multirow[b]{2}{*}{$\begin{array}{c}\text { Normalized } \\
\text { Energy Data } \\
\text { (Normalization } \\
\text { Method) }\end{array}$} & \multirow[b]{2}{*}{$\underset{\substack{\text { Magnitude } \\
\text { (in \%) }}}{\text { EPG }}$} \\
\hline & & & & $\begin{array}{l}\text { Duration } \\
\text { (Days) }\end{array}$ & $\begin{array}{c}\text { Measured } \\
\text { Energy Type } \\
\text { (Source of } \\
\text { Energy Data) }\end{array}$ & $\begin{array}{l}\text { Indoor } \\
\text { Conditions } \\
\text { (Source of } \\
\text { Data) }\end{array}$ & $\begin{array}{l}\text { Outdoor } \\
\text { Conditions } \\
\text { (Source of } \\
\text { Data) }\end{array}$ & $\begin{array}{c}\text { Observed } \\
\text { Occupant } \\
\text { Behavior } \\
\text { (Source of User } \\
\text { Behavior Data) }\end{array}$ & & \\
\hline$[17]$ & $\begin{array}{l}\text { Residential; } \\
\text { Madrid, Spain; } \\
1972\end{array}$ & $\begin{array}{c}\text { Heating, } \\
\text { cooling load } \\
\text { (simulation) }\end{array}$ & $\begin{array}{c}\text { Actual } \\
\text { occupancy } \\
\text { schedule by } \\
\text { observation }\end{array}$ & 365 & $\begin{array}{c}\text { Gas } \\
\text { consumption } \\
\text { (energy } \\
\text { metering) }\end{array}$ & $\begin{array}{c}\text { Air } \\
\text { temperature } \\
\text { (sensors) }\end{array}$ & $\begin{array}{c}\text { Air } \\
\text { temperature } \\
\text { (sensors) }\end{array}$ & $\begin{array}{l}\text { Appliances use } \\
\text { Opening } \\
\text { window } \\
\text { behavior } \\
\text { boiler set-points } \\
\text { (sensors) }\end{array}$ & NA & $\begin{array}{c}\text { Heating: } \\
-15.4 \% \text { to } \\
-5.5 \% \\
\text { cooling: }+5.1 \% \\
\text { to }+12.7 \%\end{array}$ \\
\hline [18] & $\begin{array}{c}\text { Residential; } \\
\text { Denmark; before } \\
1962 \text { until after } \\
1999\end{array}$ & $\begin{array}{l}\text { Energy } \\
\text { demand } \\
\text { (regression } \\
\text { models) }\end{array}$ & $\begin{array}{l}\text { Questionnaire } \\
\text { survey }\end{array}$ & 182 & $\begin{array}{c}\text { District } \\
\text { heating } \\
\text { (energy labels, } \\
\text { energy } \\
\text { metering } \\
\text { provided from } \\
\text { a national } \\
\text { database) }\end{array}$ & NA & NA & $\begin{array}{l}\text { Frequency of } \\
\text { adjusting } \\
\text { thermostats, } \\
\text { window } \\
\text { opening, } \\
\text { clothing during } \\
\text { winter, the } \\
\text { perceived } \\
\text { temperature } \\
\text { level compared } \\
\text { with friends } \\
\text { (questionnaire } \\
\text { survey) }\end{array}$ & NA & NA \\
\hline [19] & $\begin{array}{c}\text { Residential; } \\
\text { France; up to } 2009\end{array}$ & $\begin{array}{l}\text { Heating load } \\
\text { (The French } \\
\text { EPC, } \\
\text { simplified } \\
\text { calculation) }\end{array}$ & Survey & 365 & $\begin{array}{l}\text { Gas, fuel oil, } \\
\text { wood, } \\
\text { electricity } \\
\text { (energy bills } \\
\text { from survey) }\end{array}$ & NA & NA & NA (survey) & $\begin{array}{c}\text { Space heating } \\
\text { consumption } \\
\text { (adjusted for } \\
\text { degree days, } \\
\text { intensity factor } \\
\text { integrates } \\
\text { energy price, } \\
\text { income and } \\
\text { rebound effect } \\
\text { into a theoretical } \\
\text { budget share } \\
\text { elasticity) }\end{array}$ & $\begin{array}{c}3 \% \text { on } \\
\text { predictions if } \\
\text { EPC is } \\
\text { modified for } \\
\text { theoretical } \\
\text { budget share, } \\
\text { error } 30 \% \text { if } \\
\text { EPC is } \\
\text { modified for } \\
\text { behavioral } \\
\text { patterns. } \\
\text { Simple EPC } \\
\text { error }>65 \%\end{array}$ \\
\hline
\end{tabular}


Table 1. Cont.

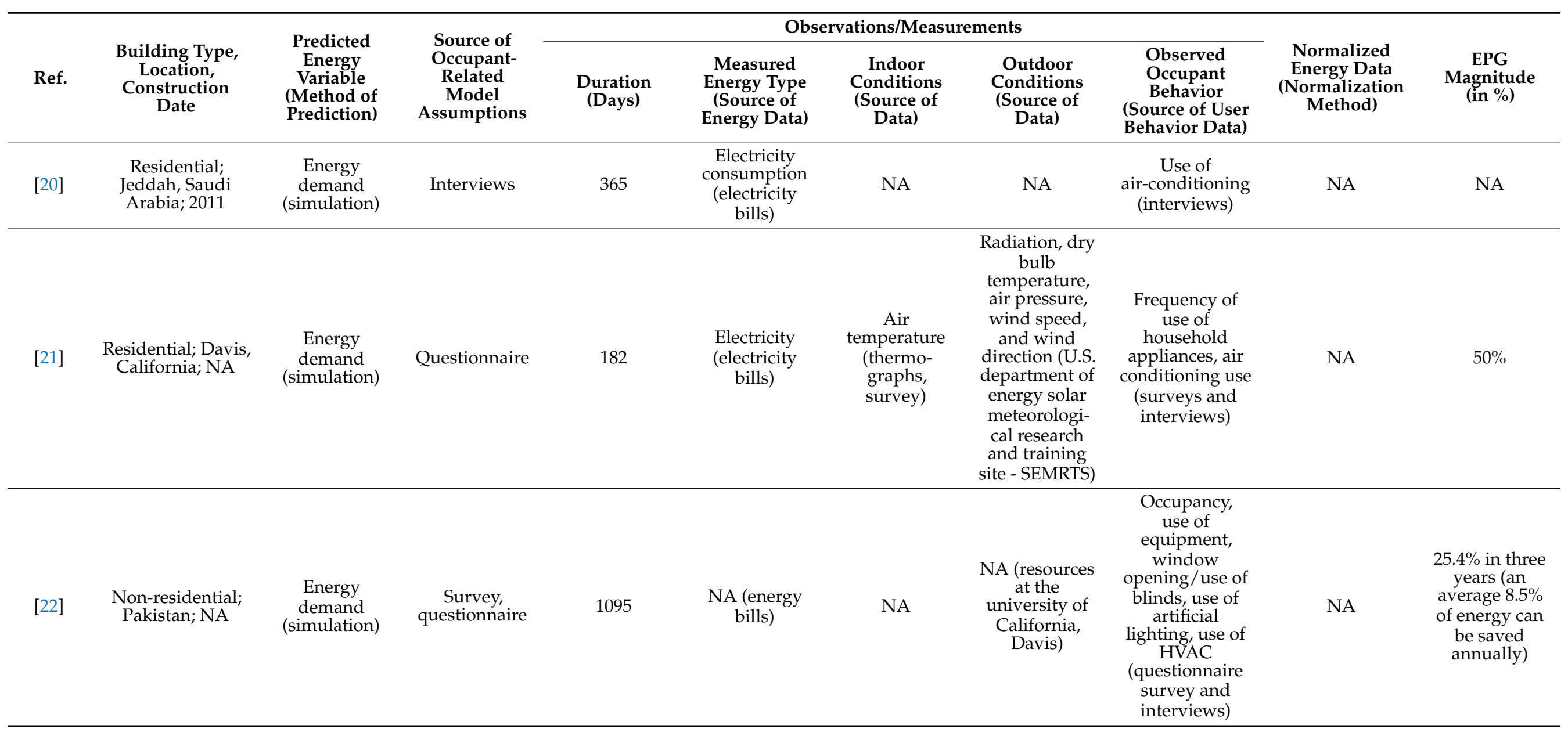


Table 1. Cont.

\begin{tabular}{|c|c|c|c|c|c|c|c|c|c|c|}
\hline \multirow[b]{2}{*}{ Ref. } & \multirow[b]{2}{*}{$\begin{array}{c}\text { Building Type, } \\
\text { Location, } \\
\text { Construction } \\
\text { Date }\end{array}$} & \multirow[b]{2}{*}{$\begin{array}{l}\text { Predicted } \\
\text { Energy } \\
\text { Variable } \\
\text { (Method of } \\
\text { Prediction) }\end{array}$} & \multirow[b]{2}{*}{$\begin{array}{l}\text { Source of } \\
\text { Occupant- } \\
\text { Related } \\
\text { Model } \\
\text { Assumptions }\end{array}$} & \multicolumn{5}{|c|}{ Observations/Measurements } & \multirow[b]{2}{*}{$\begin{array}{c}\text { Normalized } \\
\text { Energy Data } \\
\text { (Normalization } \\
\text { Method) }\end{array}$} & \multirow[b]{2}{*}{$\begin{array}{l}\text { EPG } \\
\text { Magnitude } \\
\text { (in \%) }\end{array}$} \\
\hline & & & & $\begin{array}{c}\text { Duration } \\
\text { (Days) }\end{array}$ & $\begin{array}{c}\text { Measured } \\
\text { Energy Type } \\
\text { (Source of } \\
\text { Energy Data) }\end{array}$ & $\begin{array}{l}\text { Indoor } \\
\text { Conditions } \\
\text { (Source of } \\
\text { Data) }\end{array}$ & $\begin{array}{l}\text { Outdoor } \\
\text { Conditions } \\
\text { (Source of } \\
\text { Data) }\end{array}$ & $\begin{array}{c}\text { Observed } \\
\text { Occupant } \\
\text { Behavior } \\
\text { (Source of User } \\
\text { Behavior Data) }\end{array}$ & & \\
\hline$[23]$ & $\begin{array}{l}\text { Non-residential; } \\
\text { Botswana and } \\
\text { South Africa; NA }\end{array}$ & $\begin{array}{c}\text { Energy } \\
\text { demand (NA) }\end{array}$ & Standard & 365 & $\begin{array}{l}\text { Electricity } \\
\text { consumption } \\
\text { (energy } \\
\text { auditing } \\
\text { equipment } \\
\text { (data logger)) }\end{array}$ & NA & NA & $\begin{array}{c}\text { Occupancy, use } \\
\text { of } \\
\text { air-conditioning } \\
\text { (working-hours } \\
\text { schedule, } \\
\text { interviews, site } \\
\text { inspection at } \\
\text { night) }\end{array}$ & NA & $23 \%$ to $36 \%$ \\
\hline$[24]$ & $\begin{array}{c}\text { Non-residential; } \\
\text { UK; NA }\end{array}$ & $\begin{array}{c}\text { Energy } \\
\text { demand } \\
\text { (simulation) }\end{array}$ & Standard & 2555 & $\begin{array}{l}\text { Electricity, gas } \\
\text { (utility bills) }\end{array}$ & NA & NA & $\begin{array}{c}\text { Occupancy, } \\
\text { frequency of } \\
\text { equipment use } \\
\text { (survey) }\end{array}$ & $\begin{array}{l}\text { Weather data } \\
\text { (NA) }\end{array}$ & $\begin{array}{c}\text { Electricity: } \\
175 \% \text { to } 274 \% \text {, } \\
\text { gas: } 147 \% \text { to } \\
214 \%\end{array}$ \\
\hline$[25]$ & $\begin{array}{l}\text { Residential; } \\
\text { Scotland; NA }\end{array}$ & $\begin{array}{l}\text { (A) Heating } \\
\text { load, (B) } \\
\text { energy } \\
\text { demand (SAP } \\
\text { calculation) }\end{array}$ & Standard & 365 & $\begin{array}{l}\text { (A) Gas, (B) } \\
\text { electricity } \\
\text { (energy } \\
\text { metering) }\end{array}$ & $\begin{array}{c}\text { Air } \\
\text { temperature, } \\
\text { surface } \\
\text { temperature, } \\
\text { air pressure, } \\
\text { volume flow } \\
\text { rate of air } \\
\text { (thermograms, } \\
\text { sensors) }\end{array}$ & $\begin{array}{c}\text { Air } \\
\text { temperature } \\
\text { Surface } \\
\text { temperature } \\
\text { air pressure } \\
\text { (thermograms, } \\
\text { sensors) }\end{array}$ & $\begin{array}{l}\text { Occupancy, } \\
\text { service control } \\
\text { systems, use of } \\
\text { appliances } \\
\text { (survey) }\end{array}$ & NA & $\begin{array}{c}\text { (A) control } \\
\text { house: } 42 \% \text {, } \\
\text { passive house } \\
\text { 37\%, (B) } \\
\text { control house: } \\
-43 \% \text {, passive } \\
\text { house: } 6 \%\end{array}$ \\
\hline$[26]$ & $\begin{array}{l}\text { Residential; } \\
\text { Belgium; building } \\
\text { permits of } \\
2012-2013\end{array}$ & $\begin{array}{l}\text { Heating load } \\
\text { (energy } \\
\text { certificate) }\end{array}$ & Standard & 365 & $\begin{array}{l}\text { Electricity, gas } \\
\text { (energy bills) }\end{array}$ & NA & $\begin{array}{c}\text { Air } \\
\text { temperature, } \\
\text { solar } \\
\text { irradiance } \\
\text { (national } \\
\text { climatic data) }\end{array}$ & $\begin{array}{c}\text { Occupancy, } \\
\text { heated rooms, } \\
\text { heating hours, } \\
\text { temperature } \\
\text { set-points, } \\
\text { number of } \\
\text { baths/showers } \\
\text { per week and } \\
\text { duration } \\
\text { (survey) }\end{array}$ & $\begin{array}{l}\text { Energy use for } \\
\text { space heating } \\
\text { and DHW } \\
\text { (adjusted for } \\
\text { degree days) }\end{array}$ & Gas: $-25 \%$ \\
\hline
\end{tabular}


Table 1. Cont.

\begin{tabular}{|c|c|c|c|c|c|c|c|c|c|c|}
\hline \multirow[b]{2}{*}{ Ref. } & \multirow[b]{2}{*}{$\begin{array}{c}\text { Building Type, } \\
\text { Location, } \\
\text { Construction } \\
\text { Date }\end{array}$} & \multirow[b]{2}{*}{$\begin{array}{l}\text { Predicted } \\
\text { Energy } \\
\text { Variable } \\
\text { (Method of } \\
\text { Prediction) }\end{array}$} & \multirow[b]{2}{*}{$\begin{array}{l}\text { Source of } \\
\text { Occupant- } \\
\text { Related } \\
\text { Model } \\
\text { Assumptions }\end{array}$} & \multicolumn{5}{|c|}{ Observations/Measurements } & \multirow[b]{2}{*}{$\begin{array}{c}\text { Normalized } \\
\text { Energy Data } \\
\text { (Normalization } \\
\text { Method) }\end{array}$} & \multirow[b]{2}{*}{$\begin{array}{c}\text { EPG } \\
\text { Magnitude } \\
\text { (in \%) }\end{array}$} \\
\hline & & & & $\begin{array}{c}\text { Duration } \\
\text { (Days) }\end{array}$ & $\begin{array}{c}\text { Measured } \\
\text { Energy Type } \\
\text { (Source of } \\
\text { Energy Data) }\end{array}$ & $\begin{array}{l}\text { Indoor } \\
\text { Conditions } \\
\text { (Source of } \\
\text { Data) }\end{array}$ & $\begin{array}{c}\text { Outdoor } \\
\text { Conditions } \\
\text { (Source of } \\
\text { Data) }\end{array}$ & $\begin{array}{c}\text { Observed } \\
\text { Occupant } \\
\text { Behavior } \\
\text { (Source of User } \\
\text { Behavior Data) }\end{array}$ & & \\
\hline$[27]$ & $\begin{array}{c}\text { Residential; } \\
\text { Germany; 1950s }\end{array}$ & $\begin{array}{l}\text { Heating load } \\
\text { (NA) }\end{array}$ & NA & 365 & $\begin{array}{l}\text { NA (energy } \\
\text { metering) }\end{array}$ & $\begin{array}{c}\text { Air } \\
\text { temperature, } \\
\text { relative } \\
\text { humidity, } \mathrm{CO}_{2}, \\
\text { VOC (sensors) }\end{array}$ & $\begin{array}{c}\text { Air } \\
\text { temperature, } \\
\text { solar radiation, } \\
\text { wind speed, } \\
\text { wind direction } \\
\text { (weather } \\
\text { station) }\end{array}$ & $\begin{array}{l}\text { Window use, } \\
\text { thermostat } \\
\text { settings } \\
\text { (sensors) }\end{array}$ & NA & NA \\
\hline$[28]$ & $\begin{array}{l}\text { Non-residential; } \\
\text { Germany; } \\
\text { 1920-2000 }\end{array}$ & $\begin{array}{c}\text { Heating load } \\
\text { (energy } \\
\text { certificate) }\end{array}$ & NA & $730-1825$ & $\begin{array}{l}\text { NA (on site, } \\
\text { energy } \\
\text { metering) }\end{array}$ & NA & NA & $\begin{array}{l}\text { Occupancy, } \\
\text { window } \\
\text { opening, } \\
\text { heating } \\
\text { control/set- } \\
\text { point } \\
\text { (interviews) }\end{array}$ & $\begin{array}{l}\text { Energy } \\
\text { consumption for } \\
\text { space heating } \\
\text { (weather- } \\
\text { adjusted } \\
\text { (NA)) }\end{array}$ & $\begin{array}{c}\text { Pre-retrofit: } \\
-13.8 \% \text { to } \\
2.1 \% \text {, } \\
\text { post-retrofit: } \\
-43.5 \% \text { to } \\
-8.3 \%\end{array}$ \\
\hline [29] & $\begin{array}{l}\text { Residential; } \\
\text { Netherlands; } \\
\text { 1946-1995 }\end{array}$ & NA & NA & NA & $\begin{array}{l}\text { Gas (energy } \\
\text { providers) }\end{array}$ & NA & NA & $\begin{array}{l}\text { Use of heating } \\
\text { and ventilation } \\
\text { systems (survey, } \\
\text { questionnaires) }\end{array}$ & $\begin{array}{c}\text { Thermostat } \\
\text { settings: temp } \\
\text { set-point (used } \\
\text { standard } \\
\text { deviations from } \\
\text { the mean), } \\
\text { ventilation } \\
\text { hours (consider } \\
\text { the type of } \\
\text { ventilation with } \\
\text { most hours) }\end{array}$ & NA \\
\hline$[30]$ & $\begin{array}{l}\text { Residential; UK; } \\
\text { NA }\end{array}$ & $\begin{array}{c}\text { Energy } \\
\text { demand (SAP } \\
\text { calculation) }\end{array}$ & Standard & NA & $\begin{array}{l}\text { NA (energy } \\
\text { metering) }\end{array}$ & NA & NA & $\begin{array}{c}\text { Occupancy } \\
\text { patterns (NA) }\end{array}$ & NA & $60 \%$ \\
\hline$[31]$ & $\begin{array}{c}\text { Residential; UK; } \\
1930\end{array}$ & $\begin{array}{c}\text { Energy } \\
\text { demand } \\
\text { (simulation) }\end{array}$ & Survey & 30 & $\begin{array}{c}\text { Gas, electricity } \\
\text { (energy } \\
\text { metering) }\end{array}$ & $\begin{array}{c}\text { Air } \\
\text { temperature } \\
\text { (Hobo } \\
\text { sensors) }\end{array}$ & $\begin{array}{c}\text { Air } \\
\text { temperature, } \\
\text { irradiance } \\
\text { (weather } \\
\text { station } 20 \mathrm{~km} \\
\text { away) }\end{array}$ & $\begin{array}{l}\text { Temperature } \\
\text { set-points } \\
\text { (sensors) }\end{array}$ & NA & $-14 \%$ to $74 \%$ \\
\hline
\end{tabular}


Table 1. Cont.

\begin{tabular}{|c|c|c|c|c|c|c|c|c|c|c|}
\hline \multirow[b]{2}{*}{ Ref. } & \multirow[b]{2}{*}{$\begin{array}{l}\text { Building Type, } \\
\text { Location, } \\
\text { Construction } \\
\text { Date }\end{array}$} & \multirow[b]{2}{*}{$\begin{array}{l}\text { Predicted } \\
\text { Energy } \\
\text { Variable } \\
\text { (Method of } \\
\text { Prediction) }\end{array}$} & \multirow[b]{2}{*}{$\begin{array}{c}\text { Source of } \\
\text { Occupant- } \\
\text { Related } \\
\text { Model } \\
\text { Assumptions }\end{array}$} & \multicolumn{5}{|c|}{ Observations/Measurements } & \multirow[b]{2}{*}{$\begin{array}{c}\text { Normalized } \\
\text { Energy Data } \\
\text { (Normalization } \\
\text { Method) }\end{array}$} & \multirow[b]{2}{*}{$\begin{array}{c}\text { EPG } \\
\text { Magnitude } \\
\text { (in \%) }\end{array}$} \\
\hline & & & & $\begin{array}{c}\text { Duration } \\
\text { (Days) }\end{array}$ & $\begin{array}{c}\text { Measured } \\
\text { Energy Type } \\
\text { (Source of } \\
\text { Energy Data) }\end{array}$ & $\begin{array}{l}\text { Indoor } \\
\text { Conditions } \\
\text { (Source of } \\
\text { Data) }\end{array}$ & $\begin{array}{l}\text { Outdoor } \\
\text { Conditions } \\
\text { (Source of } \\
\text { Data) }\end{array}$ & $\begin{array}{c}\text { Observed } \\
\text { Occupant } \\
\text { Behavior } \\
\text { (Source of User } \\
\text { Behavior Data) }\end{array}$ & & \\
\hline$[32]$ & $\begin{array}{c}\text { Residential; UK; } \\
\text { NA }\end{array}$ & $\begin{array}{c}\text { NA (SAP } \\
\text { calculations) }\end{array}$ & Codes & 365 & $\begin{array}{l}\text { Electricity } \\
\text { (energy } \\
\text { metering) }\end{array}$ & NA (sensors) & $\begin{array}{c}\text { Air } \\
\text { temperature } \\
\text { (sensors) }\end{array}$ & $\begin{array}{c}\text { Window } \\
\text { opening, } \\
\text { temperature } \\
\text { set-point (NA) }\end{array}$ & NA & NA \\
\hline$[34]$ & $\begin{array}{l}\text { Non-residential; } \\
\text { Switzerland; NA }\end{array}$ & $\begin{array}{c}\text { Lighting load } \\
\text { (simulation) }\end{array}$ & Standard & 2920 & $\begin{array}{c}\text { Electric } \\
\text { lighting } \\
\text { energy (energy } \\
\text { metering) }\end{array}$ & $\begin{array}{c}\text { Air } \\
\text { temperature, } \\
\text { Illuminance, } \\
\text { luminance } \\
\text { (sensors) }\end{array}$ & NA & $\begin{array}{l}\text { Occupancy, } \\
\text { blinds position, } \\
\text { electric lighting } \\
\text { behavior } \\
\text { (sensors) }\end{array}$ & $\begin{array}{l}\text { Electric lighting } \\
\text { consumption } \\
\text { (consider the } \\
\text { impact of } \\
\text { different } \\
\text { occupancy rates) }\end{array}$ & $\begin{array}{c}\text { Advanced } \\
\text { controller: } 72 \% \\
\text { (simulation), } \\
\text { "Best practice" } \\
\text { controller: 19\% } \\
\text { (actual) }\end{array}$ \\
\hline [35] & $\begin{array}{l}\text { Non-residential; } \\
\text { UK; 2001-2005 }\end{array}$ & $\begin{array}{c}\text { Energy } \\
\text { demand } \\
\text { (CIBSE } \\
\text { calculation) }\end{array}$ & $\begin{array}{c}\text { Codes (CIBSE, } \\
\text { BB87, SAP) }\end{array}$ & NA & $\begin{array}{c}\text { Electricity, } \\
\text { natural gas } \\
\text { (energy } \\
\text { metering) }\end{array}$ & NA & $\begin{array}{c}\text { Air } \\
\text { temperature } \\
\text { (local weather } \\
\text { office) }\end{array}$ & $\begin{array}{l}\text { Lighting control } \\
\text { ON/OFF status } \\
\text { (observations } \\
\text { and sensors) }\end{array}$ & NA & $57 \%$ to $66 \%$ \\
\hline$[36]$ & $\begin{array}{l}\text { Non-residential; } \\
\text { Canada; NA }\end{array}$ & $\begin{array}{c}\text { Energy } \\
\text { demand } \\
\text { (simulation) }\end{array}$ & $\begin{array}{l}\text { Standard, } \\
\text { database }\end{array}$ & 365 & $\begin{array}{l}\text { Electricity } \\
\text { (energy } \\
\text { metering) }\end{array}$ & $\begin{array}{c}\text { Air } \\
\text { temperature, } \\
\text { set-points, } \\
\mathrm{CO}_{2} \\
\text { Total Volatile } \\
\text { Organic } \\
\text { Compounds } \\
\text { (TVOC) } \\
\text { (sensors) }\end{array}$ & $\begin{array}{l}\text { Weather } \\
\text { (sensors) }\end{array}$ & $\begin{array}{l}\text { Occupancy, } \\
\text { window status, } \\
\text { temperature } \\
\text { set-points } \\
\text { (sensors) }\end{array}$ & NA & $60 \%$ \\
\hline
\end{tabular}


Table 1. Cont.

\begin{tabular}{|c|c|c|c|c|c|c|c|c|c|c|}
\hline \multirow[b]{2}{*}{ Ref. } & \multirow[b]{2}{*}{$\begin{array}{l}\text { Building Type, } \\
\text { Location, } \\
\text { Construction } \\
\text { Date }\end{array}$} & \multirow[b]{2}{*}{$\begin{array}{l}\text { Predicted } \\
\text { Energy } \\
\text { Variable } \\
\text { (Method of } \\
\text { Prediction) }\end{array}$} & \multirow[b]{2}{*}{$\begin{array}{l}\text { Source of } \\
\text { Occupant- } \\
\text { Related } \\
\text { Model } \\
\text { Assumptions }\end{array}$} & \multicolumn{5}{|c|}{ Observations/Measurements } & \multirow[b]{2}{*}{$\begin{array}{c}\text { Normalized } \\
\text { Energy Data } \\
\text { (Normalization } \\
\text { Method) }\end{array}$} & \multirow[b]{2}{*}{$\underset{\substack{\text { Magnitude } \\
\text { (in \%) }}}{\text { EPG }}$} \\
\hline & & & & $\begin{array}{c}\text { Duration } \\
\text { (Days) }\end{array}$ & $\begin{array}{c}\text { Measured } \\
\text { Energy Type } \\
\text { (Source of } \\
\text { Energy Data) }\end{array}$ & $\begin{array}{l}\text { Indoor } \\
\text { Conditions } \\
\text { (Source of } \\
\text { Data) }\end{array}$ & $\begin{array}{c}\text { Outdoor } \\
\text { Conditions } \\
\text { (Source of } \\
\text { Data) }\end{array}$ & $\begin{array}{c}\text { Observed } \\
\text { Occupant } \\
\text { Behavior } \\
\text { (Source of User } \\
\text { Behavior Data) }\end{array}$ & & \\
\hline [37] & $\begin{array}{c}\text { Residential; } \\
\text { Munich, Germany; } \\
2010\end{array}$ & $\begin{array}{l}\text { Heating load } \\
\text { (code/norm) }\end{array}$ & $\begin{array}{l}\text { Standard (DIN } \\
\text { V 4108-6 } \\
\text { (EnEV 2009)) }\end{array}$ & 365 & $\begin{array}{l}\text { Electricity, } \\
\text { other (energy } \\
\text { metering) }\end{array}$ & $\begin{array}{c}\text { Air } \\
\text { temperature } \\
\text { (sensors) }\end{array}$ & $\begin{array}{c}\text { Air } \\
\text { temperature, } \\
\text { global } \\
\text { radiation } \\
\text { (sensors) }\end{array}$ & $\begin{array}{l}\text { Window } \\
\text { opening } \\
\text { frequency } \\
\text { (sensors) }\end{array}$ & $\begin{array}{l}\text { Heating energy } \\
\text { (additional } \\
\text { component from } \\
\text { DHW (60\% } \\
\text { losses); weather } \\
\text { adjusted) }\end{array}$ & $\begin{array}{l}\text { Building: } \\
\text { +15\%; } \\
\text { apartment: } \\
-59 \% \text { to } \\
+318 \%\end{array}$ \\
\hline$[38]$ & $\begin{array}{l}\text { Non-residential; } \\
\text { USA; NA }\end{array}$ & $\begin{array}{c}\text { Energy } \\
\text { demand } \\
\text { (simulation) }\end{array}$ & Standard & 3304 & $\begin{array}{l}\text { Electricity } \\
\text { (energy } \\
\text { metering) }\end{array}$ & NA & NA & $\begin{array}{l}\text { Occupancy } \\
\text { patterns (IR and } \\
\text { video-based } \\
\text { sensors) }\end{array}$ & NA & $9 \%$ \\
\hline [39] & $\begin{array}{l}\text { Residential and } \\
\text { Non-residential; } \\
\text { Canada; NA }\end{array}$ & $\begin{array}{c}\text { Energy } \\
\text { demand } \\
\text { (simulation) }\end{array}$ & Standard & 2555 & $\begin{array}{l}\text { Electricity } \\
\text { (utility bills) }\end{array}$ & $\begin{array}{l}\text { Illuminance } \\
(\mathrm{NA})\end{array}$ & NA & $\begin{array}{l}\text { Occupancy } \\
\text { (survey) }\end{array}$ & NA & $0 \%$ to $69 \%$ \\
\hline$[40]$ & $\begin{array}{l}\text { Residential; } \\
\text { Belgium; 1960s }\end{array}$ & $\begin{array}{l}\text { Heating load } \\
\text { (quasi-steady } \\
\text { state model } \\
\text { calculation) }\end{array}$ & Standard & 365 & $\begin{array}{l}\text { Gas (energy } \\
\text { metering) }\end{array}$ & $\begin{array}{c}\text { Heat flux, air } \\
\text { tightness, } \mathrm{CO}_{2} \\
\text { air } \\
\text { temperature, } \\
\text { relative } \\
\text { humidity } \\
\text { (sensors) }\end{array}$ & $\begin{array}{c}\text { Air } \\
\text { temperature } \\
\text { (NA) }\end{array}$ & $\begin{array}{l}\text { Occupancy } \\
\text { (surveys) }\end{array}$ & $\begin{array}{c}\text { Gas } \\
\text { consumption } \\
\text { (adjusted for } \\
\text { degree days); } \\
\text { Set-point } \\
\text { temperatures } \\
\text { (deducted from } \\
\text { the average } \\
\text { point of } \\
\text { stabilization of } \\
\text { the indoor } \\
\text { temperature } \\
\text { during } \\
\text { occupancy) }\end{array}$ & $\begin{array}{c}\text { Base model: } \\
-25 \% \text { to } \\
-75 \%, \\
\text { (average } \\
-46 \% \text { ), } \\
\text { calibration: } 0 \% \\
\text { to }-75 \% \text {, } \\
\text { (average: } \\
-43 \% \text { ) }\end{array}$ \\
\hline$[41]$ & $\begin{array}{l}\text { Non-residential; } \\
\text { USA; } 2010\end{array}$ & NA & NA & 365 & $\begin{array}{c}\text { Energy } \\
\text { demand } \\
\text { (energy } \\
\text { metering) }\end{array}$ & NA & NA & $\begin{array}{c}\text { Occupancy, } \\
\text { equipment } \\
\text { usage (sensors) }\end{array}$ & NA & $40 \%$ (HVAC) \\
\hline
\end{tabular}


Table 1. Cont.

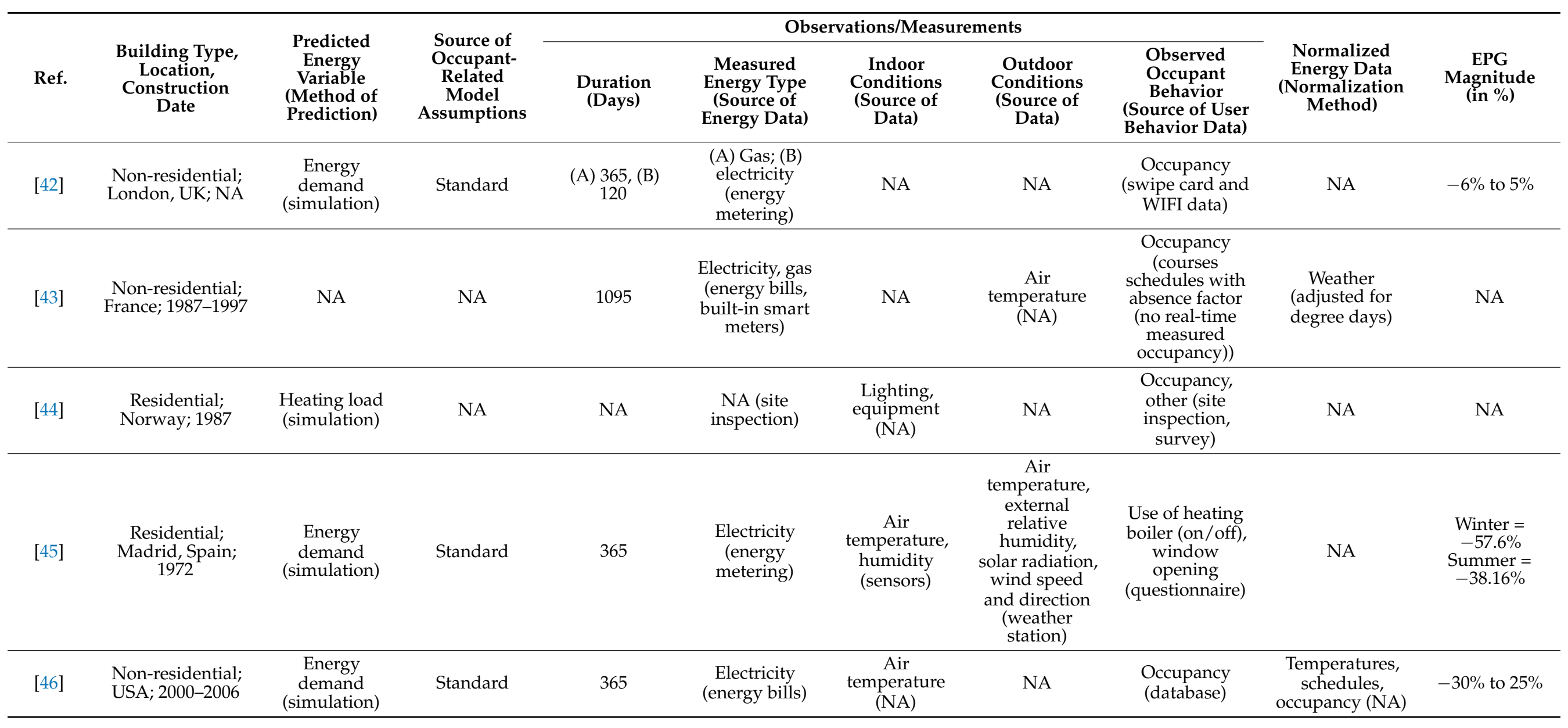


Table 1. Cont.

\begin{tabular}{|c|c|c|c|c|c|c|c|c|c|c|}
\hline \multirow[b]{2}{*}{ Ref. } & \multirow[b]{2}{*}{$\begin{array}{c}\text { Building Type, } \\
\text { Location, } \\
\text { Construction } \\
\text { Date }\end{array}$} & \multirow[b]{2}{*}{$\begin{array}{l}\text { Predicted } \\
\text { Energy } \\
\text { Variable } \\
\text { (Method of } \\
\text { Prediction) }\end{array}$} & \multirow[b]{2}{*}{$\begin{array}{l}\text { Source of } \\
\text { Occupant- } \\
\text { Related } \\
\text { Model } \\
\text { Assumptions }\end{array}$} & \multicolumn{5}{|c|}{ Observations/Measurements } & \multirow[b]{2}{*}{$\begin{array}{c}\text { Normalized } \\
\text { Energy Data } \\
\text { (Normalization } \\
\text { Method) }\end{array}$} & \multirow[b]{2}{*}{$\underset{\substack{\text { Magnitude } \\
\text { (in \%) }}}{\text { EPG }}$} \\
\hline & & & & $\begin{array}{c}\text { Duration } \\
\text { (Days) }\end{array}$ & $\begin{array}{c}\text { Measured } \\
\text { Energy Type } \\
\text { (Source of } \\
\text { Energy Data) }\end{array}$ & $\begin{array}{l}\text { Indoor } \\
\text { Conditions } \\
\text { (Source of } \\
\text { Data) }\end{array}$ & $\begin{array}{l}\text { Outdoor } \\
\text { Conditions } \\
\text { (Source of } \\
\text { Data) }\end{array}$ & $\begin{array}{c}\text { Observed } \\
\text { Occupant } \\
\text { Behavior } \\
\text { (Source of User } \\
\text { Behavior Data) }\end{array}$ & & \\
\hline [47] & $\begin{array}{l}\text { Non-residential; } \\
\text { Spain; } 2016\end{array}$ & $\begin{array}{l}\text { Energy } \\
\text { demand } \\
\text { (energy } \\
\text { certificate) }\end{array}$ & $\begin{array}{l}\text { Standard } \\
\text { (Spanish } \\
\text { building } \\
\text { regulations) }\end{array}$ & NA & $\begin{array}{c}\text { Natural gas, } \\
\text { electricity } \\
\text { (energy bills) }\end{array}$ & NA & NA & $\begin{array}{c}\text { Office } \\
\text { equipment } \\
\text { operation } \\
\text { (on/off) when } \\
\text { not in use (site } \\
\text { visits and } \\
\text { informal user } \\
\text { interviews) }\end{array}$ & NA & $30 \%$ (average) \\
\hline [48] & $\begin{array}{l}\text { Residential; } \\
\text { Sweden; NA }\end{array}$ & $\begin{array}{c}\text { Energy } \\
\text { demand } \\
\text { (simulation) }\end{array}$ & NA & 80 & NA & $\begin{array}{c}\text { Air } \\
\text { temperature } \\
\text { (NA) }\end{array}$ & $\begin{array}{c}\text { Air } \\
\text { temperature } \\
\text { (NA) }\end{array}$ & $\begin{array}{c}\text { Occupancy } \\
\text { (observations) }\end{array}$ & NA & $13.30 \%$ \\
\hline [49] & $\begin{array}{c}\text { Residential; } \\
\text { Germany; 1950s }\end{array}$ & $\begin{array}{c}\text { Energy } \\
\text { demand } \\
\text { (calculation) }\end{array}$ & NA & 1460 & $\begin{array}{c}\text { Electricity, gas, } \\
\text { district } \\
\text { heating } \\
\text { (energy } \\
\text { metering) }\end{array}$ & $\begin{array}{c}\text { Air } \\
\text { temperature } \\
\text { relative } \\
\text { humidity, } \\
\mathrm{CO}_{2} \text {, volatile } \\
\text { organic } \\
\text { compounds } \\
\text { (VOC), light } \\
\text { on the ceiling } \\
\text { (Lux), in- } \\
\text { frared/visible } \\
\text { light ratio (to } \\
\text { recognize the } \\
\text { light source), } \\
\text { (sensors) }\end{array}$ & NA & $\begin{array}{l}\text { Occupancy, } \\
\text { window } \\
\text { opening } \\
\text { (open/closed) } \\
\text { (sensors) }\end{array}$ & $\begin{array}{c}\text { Indoor air } \\
\text { temperature } \\
\text { (corrected by } \\
-2.5 \mathrm{~K})\end{array}$ & $41 \%$ to $117 \%$ \\
\hline
\end{tabular}


Table 1. Cont.

\begin{tabular}{|c|c|c|c|c|c|c|c|c|c|c|}
\hline \multirow[b]{2}{*}{ Ref. } & \multirow[b]{2}{*}{$\begin{array}{l}\text { Building Type, } \\
\text { Location, } \\
\text { Construction } \\
\text { Date }\end{array}$} & \multirow[b]{2}{*}{$\begin{array}{l}\text { Predicted } \\
\text { Energy } \\
\text { Variable } \\
\text { (Method of } \\
\text { Prediction) }\end{array}$} & \multirow[b]{2}{*}{$\begin{array}{l}\text { Source of } \\
\text { Occupant- } \\
\text { Related } \\
\text { Model } \\
\text { Assumptions }\end{array}$} & \multicolumn{5}{|c|}{ Observations/Measurements } & \multirow[b]{2}{*}{$\begin{array}{c}\text { Normalized } \\
\text { Energy Data } \\
\text { (Normalization } \\
\text { Method) }\end{array}$} & \multirow[b]{2}{*}{$\begin{array}{l}\text { EPG } \\
\text { Magnitude } \\
\text { (in \%) }\end{array}$} \\
\hline & & & & $\begin{array}{c}\text { Duration } \\
\text { (Days) }\end{array}$ & $\begin{array}{c}\text { Measured } \\
\text { Energy Type } \\
\text { (Source of } \\
\text { Energy Data) }\end{array}$ & $\begin{array}{c}\text { Indoor } \\
\text { Conditions } \\
\text { (Source of } \\
\text { Data) }\end{array}$ & $\begin{array}{c}\text { Outdoor } \\
\text { Conditions } \\
\text { (Source of } \\
\text { Data) }\end{array}$ & $\begin{array}{c}\text { Observed } \\
\text { Occupant } \\
\text { Behavior } \\
\text { (Source of User } \\
\text { Behavior Data) }\end{array}$ & & \\
\hline$[50]$ & $\begin{array}{l}\text { Non-residential; } \\
\text { Odense, Denmark; } \\
2015\end{array}$ & $\begin{array}{c}\text { Energy } \\
\text { demand } \\
\text { (simulation) }\end{array}$ & Standard & 120 & $\begin{array}{l}\text { Electricity, } \\
\text { other (energy } \\
\text { metering) }\end{array}$ & $\begin{array}{c}\text { Air } \\
\text { temperature, } \\
\text { humidity, } \mathrm{CO}_{2}, \\
\text { PIR, } \\
\text { illuminance, } \\
\text { radiator valve } \\
\text { position, } \\
\text { ventilation } \\
\text { damper } \\
\text { position, } \\
\text { blinds position } \\
\text { and multiple } \\
\text { temperature } \\
\text { and pressure } \\
\text { along the } \\
\text { energy } \\
\text { supply scheme } \\
\text { (sensors) }\end{array}$ & $\begin{array}{c}\text { Air } \\
\text { temperature, } \\
\text { wind speed, } \\
\text { solar } \\
\text { irradiation } \\
\text { (local weather } \\
\text { station) }\end{array}$ & $\begin{array}{l}\text { Occupancy } \\
\text { (cameras) }\end{array}$ & NA & $\begin{array}{c}\text { Calibrated } \\
\text { model vs. } \\
\text { measured } \\
\text { Heating: }-8.4 \\
\text { to } 6.9, \text { annual } \\
\text { regulatory } \\
\text { performance } \\
\text { vs. design } \\
\text { certificates: } \\
27.2 \% \text { to } \\
\text { 122.3\%, overal } \\
\text { dynamic } \\
\text { energy } \\
\text { performance } \\
\text { gap }-3.47 \% \text { to } \\
5.48 \%\end{array}$ \\
\hline [51] & $\begin{array}{l}\text { Non-residential; } \\
\text { London, UK; NA }\end{array}$ & $\begin{array}{c}\text { Energy } \\
\text { demand } \\
\text { (simulation) }\end{array}$ & $\begin{array}{l}\text { Standard } \\
\text { occupancy } \\
\text { profiles }\end{array}$ & NA & $\begin{array}{l}\text { Electricity } \\
\text { (energy } \\
\text { Metering) }\end{array}$ & NA & NA & $\begin{array}{c}\text { Use of } \\
\text { appliances and } \\
\text { equipment, } \\
\text { occupancy } \\
\text { (sensor) }\end{array}$ & NA & $18 \%$ \\
\hline [52] & $\begin{array}{l}\text { Residential; } \\
\text { Norway; } 1987\end{array}$ & $\begin{array}{c}\text { Heating load } \\
\text { (simulation) }\end{array}$ & Standard & 365 & $\begin{array}{l}\text { Electricity } \\
\text { (energy } \\
\text { metering) }\end{array}$ & $\begin{array}{c}\text { Air } \\
\text { temperature, } \\
\text { mechanical } \\
\text { ventilation } \\
\text { rate (sensor) }\end{array}$ & $\begin{array}{l}\text { Weather } \\
\text { (weather } \\
\text { station) }\end{array}$ & $\begin{array}{c}\text { Heating } \\
\text { set-point (visual } \\
\text { inspection) }\end{array}$ & NA & $50 \%$ \\
\hline [53] & $\begin{array}{l}\text { Residential; UK; } \\
2012\end{array}$ & $\begin{array}{c}\text { Energy } \\
\text { demand (SAP } \\
\text { predictions, } \\
\text { spreadsheet } \\
\text { calculations) }\end{array}$ & Standard & 365 & $\begin{array}{l}\text { Electricity } \\
\text { (Ewgeco } \\
\text { energy } \\
\text { monitoring } \\
\text { devices) }\end{array}$ & $\begin{array}{c}\text { Air } \\
\text { temperature, } \\
\mathrm{RH}^{2} \text { and } \mathrm{CO}_{2} \\
\text { (wireless } \\
\text { RT:Wi5 } \\
\text { data-hub) }\end{array}$ & NA & $\begin{array}{l}\text { Operation of } \\
\text { thermostats } \\
\text { (surveys and } \\
\text { interview) }\end{array}$ & NA & $180 \%$ to $250 \%$ \\
\hline
\end{tabular}


Table 1. Cont.

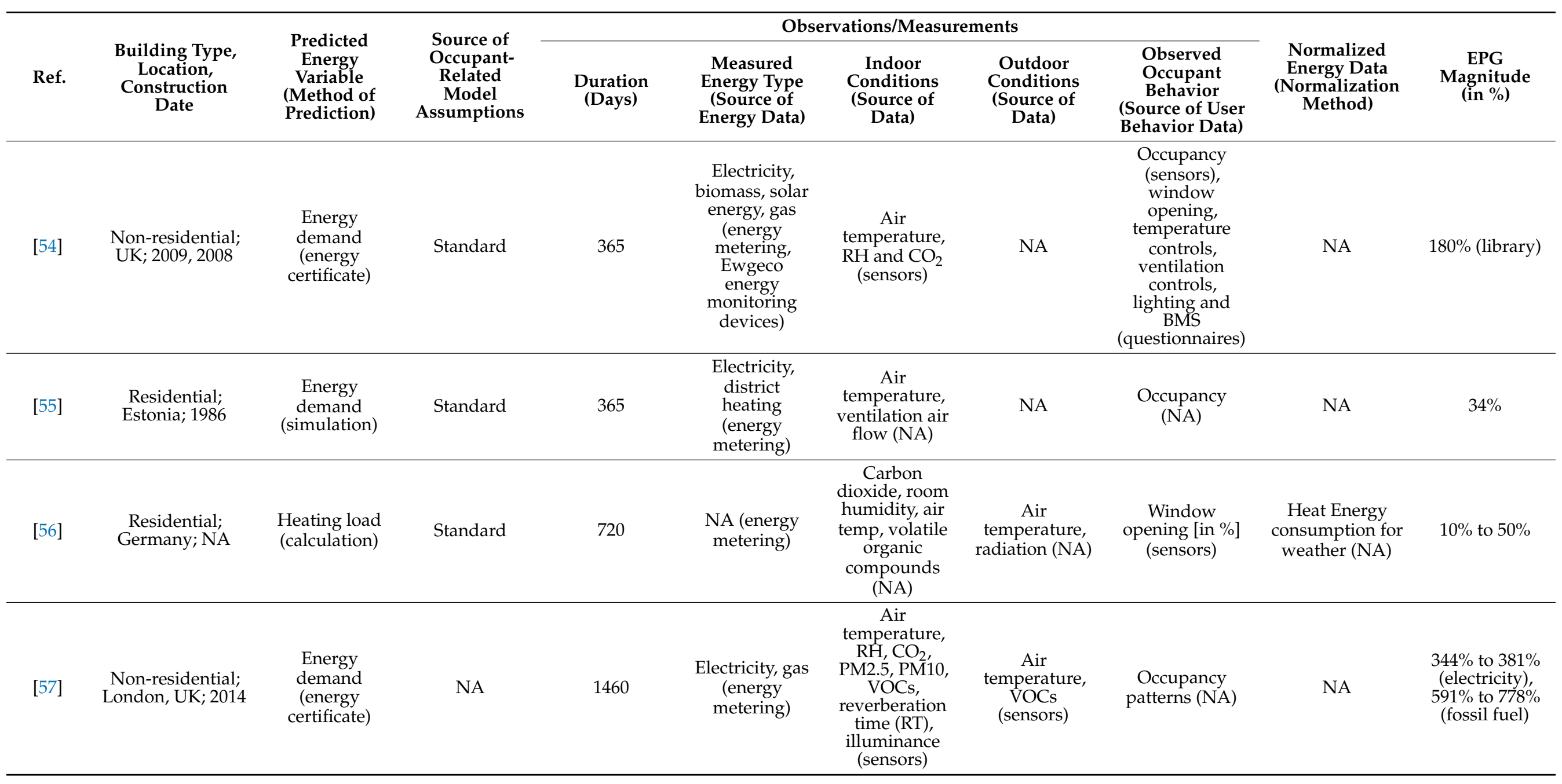


Table 1. Cont.

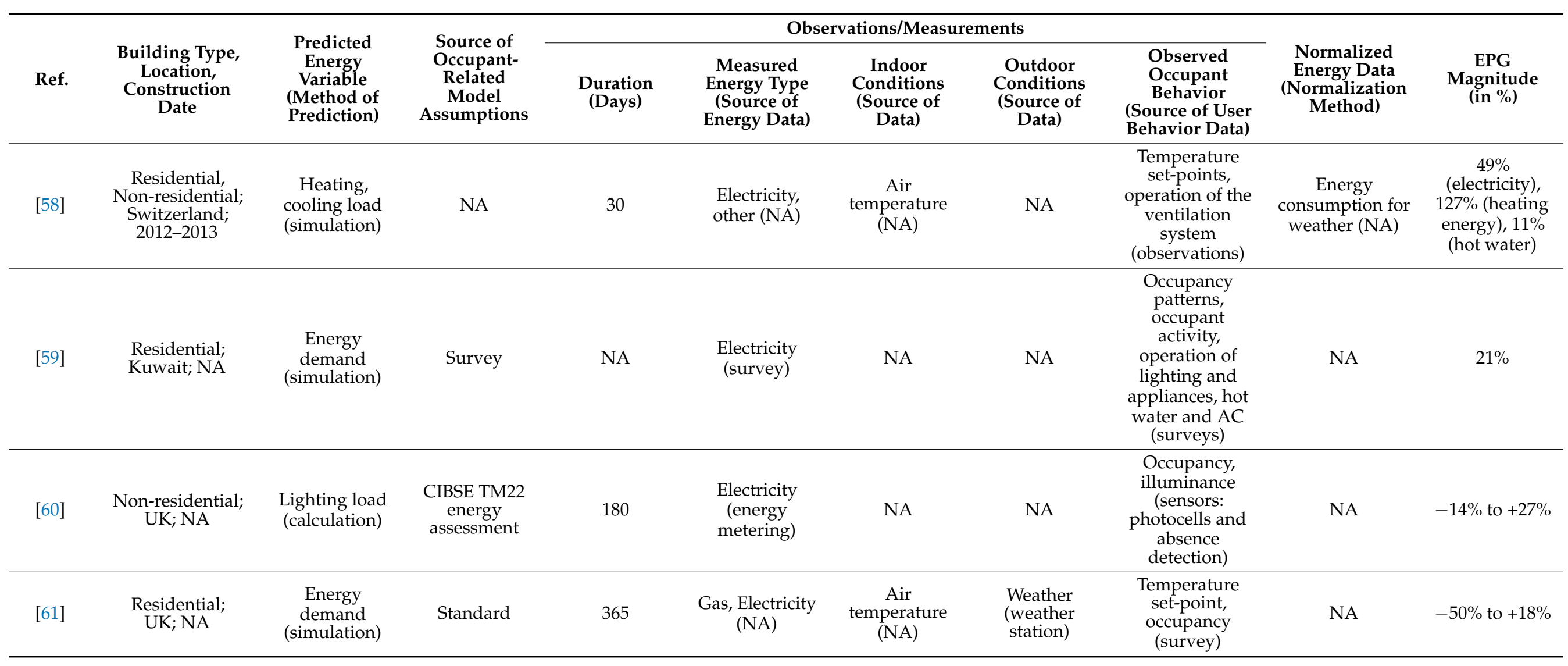


After systematically reviewing studies on the EPG, we focused on those papers that had provided quantitative evidence when suggesting that the performance gap is caused by OB. To this end, studies that have empirical measures of both occupant and non-occupant related causes of the performance gap were considered particularly relevant to the aim of this review.

\subsection{Synthesis}

Subsequent to the selection process, the papers were reviewed to extract details of the relevant geographical area, the building-related data (e.g., typology, project details), and occupant-related information (e.g., number of people, household composition, age). Second, the methods applied to predict and measure the performance gap were investigated. In terms of measured energy performance, the characteristics of the empirical data used (temporal and spatial granularity) and the data sources (sensors, records) were taken into consideration. In terms of predicted energy performance, the applied methods (e.g., energy certificates, energy simulation) and the assumptions concerning OB were extracted, analyzed, and synthesized. Finally, the methods used to identify the causes of the performance gap were investigated. Potential solutions to bridge the performance gap were discussed to address methods for and inconsistencies in the prediction and measurement of building energy performances and analysis methods of performance gaps.

\section{Review Results}

\subsection{Overview}

The vast majority of the studies $(90 \%)$ mentioned in this review were published after 2010. Only a few papers (10\%) were published prior to 2010 (Figure 2). Specifically, the scientific production in the 2015 to 2020 period was twice as high as the preceding five-year period (2010-2015). Most of the papers were published in the journals "Energy and Buildings" (33\%) and "Building and Environment" (10\%). The most frequently used words in the papers' titles were as follows: energy (53), performance (43), building or buildings (33), gap (20), consumption (10), actual (9), analysis (8), occupant (8), evaluation (7), residential (7), impact (7). Figure 3 illustrates the most frequently used words in the papers' titles as well as the frequency of included key words.

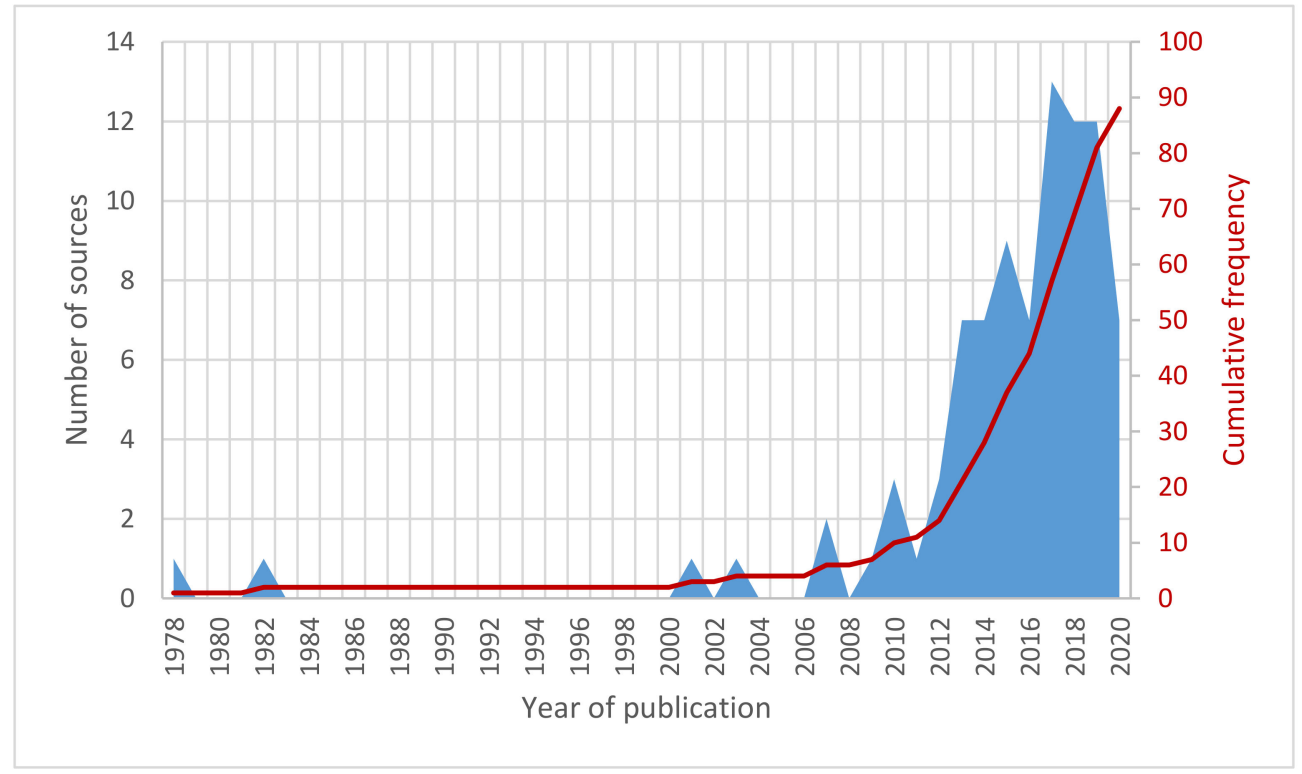

Figure 2. Distribution of the reviewed articles with regard to publication year. 


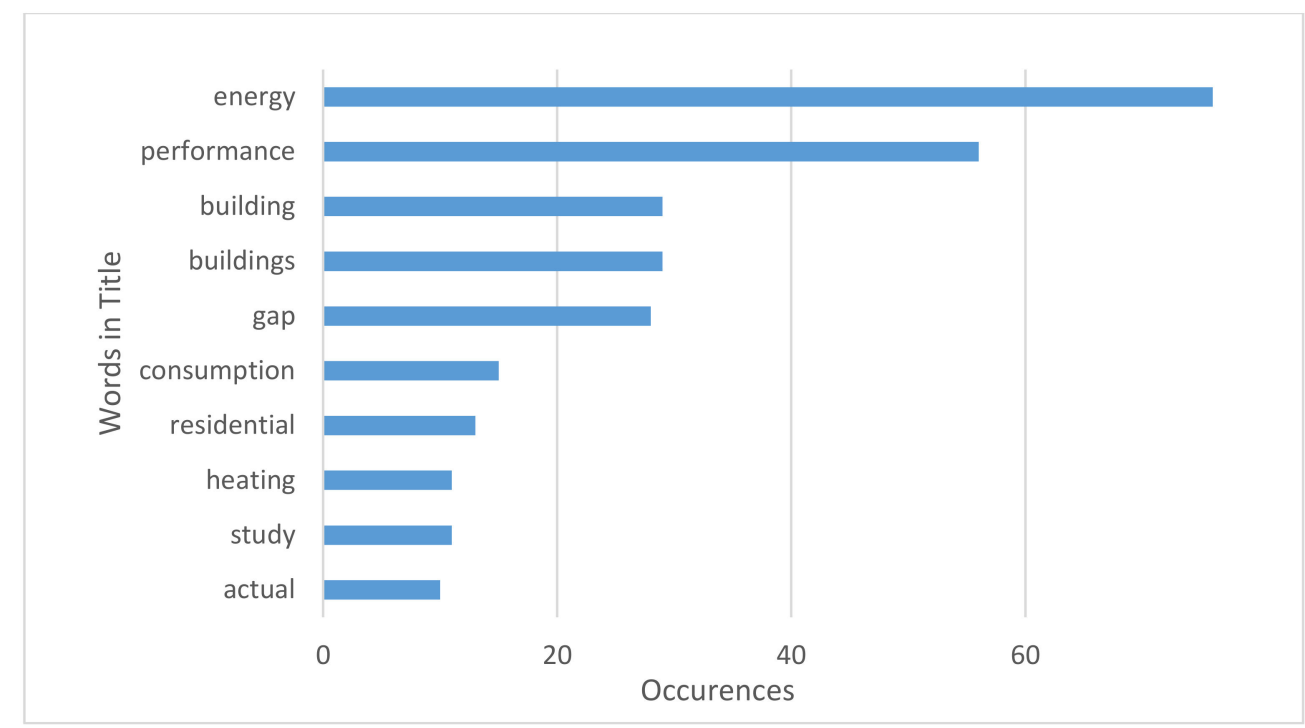

(a)

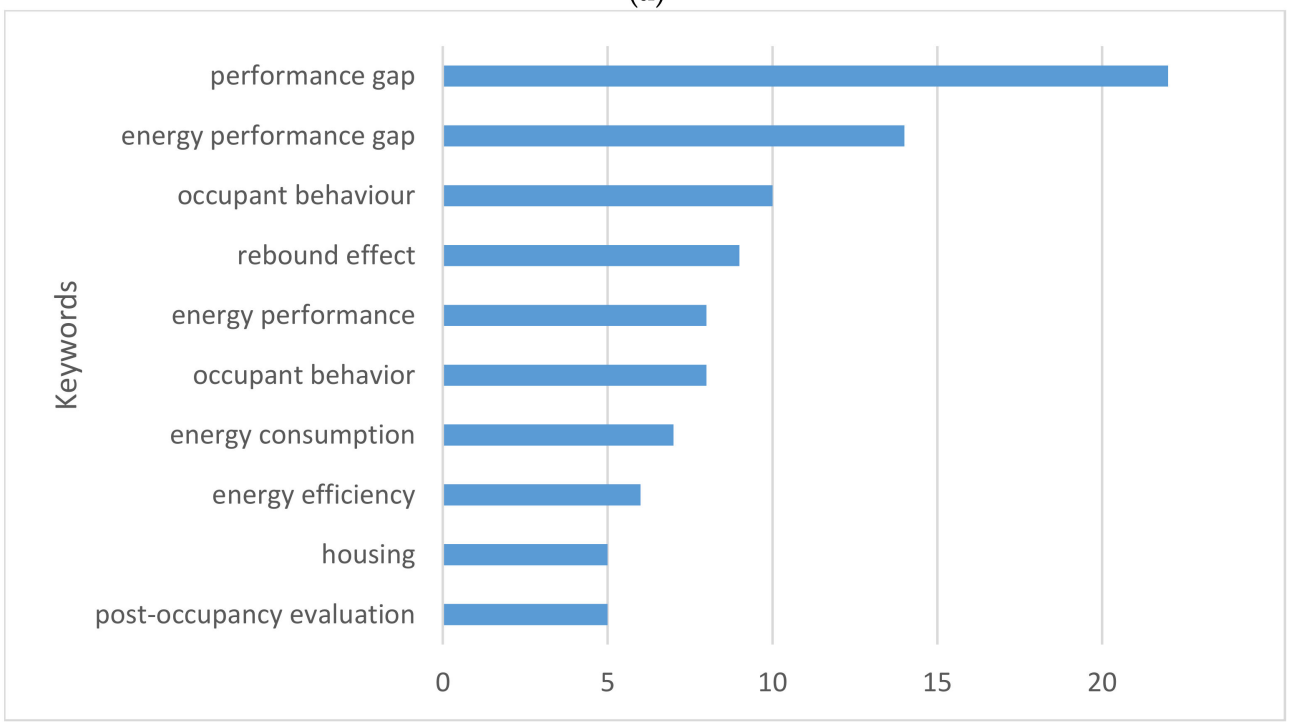

(b)

Figure 3. Frequency of relevant terms in papers' titles (a) as well as used keywords' frequency (b).

\subsection{Basic Characteristics of the Studies' Objects}

This review encompasses studies from 26 different countries (Figure 4). The vast majority $(78 \%)$ of the studies include data gathered in Europe, with the largest number of studies from the United Kingdom (25). Other studies originated from the United States, Canada, Iran, Pakistan, Saudi Arabia, Kuwait, China, South Korea, Hong Kong, Australia, South Africa and Botswana. Most studies were conducted in temperate climates. A few studies were conducted in an arid climate with very dry and hot summers (Australia, Saudi Arabia, Kuwait, Pakistan, Iran and Botswana), a subtropical climate with hot and wet summers (Hong Kong, China, South Africa) and a Mediterranean climate with hot, dry summers and cool, wet winters (Italy, Greece, South Africa). Most studies were conducted in Western countries. As such, other building contexts, related lifestyles, and occupant densities appear to be under-researched. 


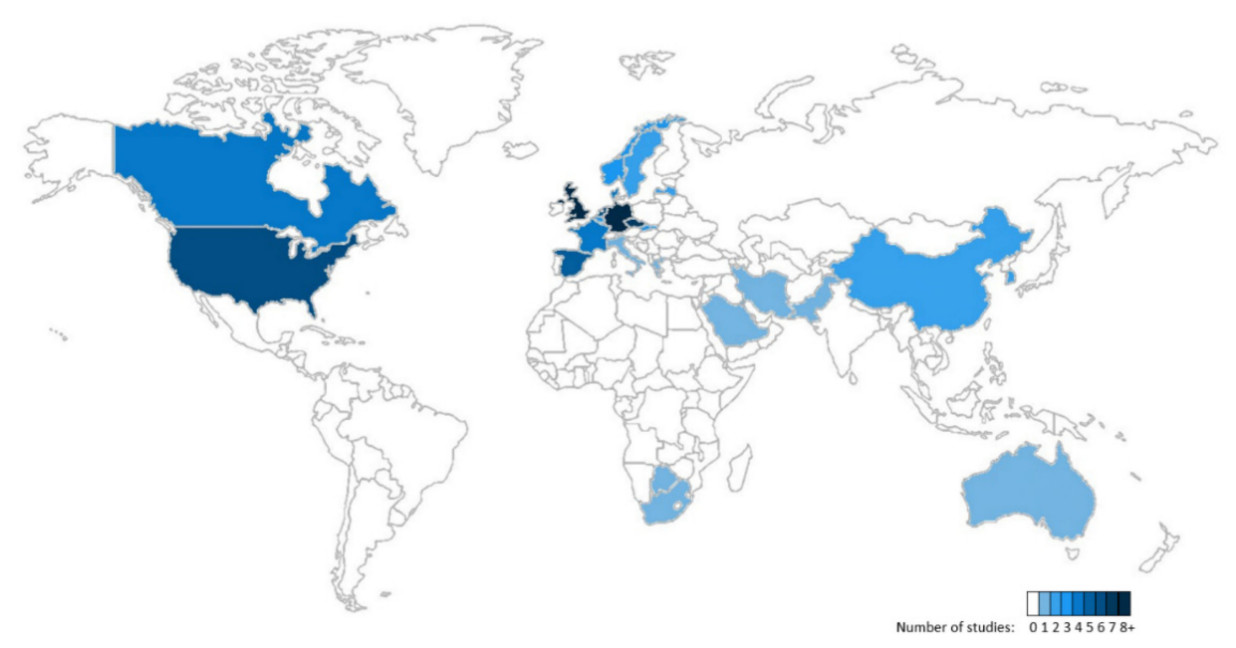

Figure 4. Illustration of the location/number of the studies.

Almost $60 \%$ of the studies investigated residential buildings. Other typologies investigated were offices [51,62-65], educational buildings [8,47,50,62,66-69], and other building types such as laboratories [41,70]. However, for non-residential buildings, very little additional information was available beyond the basic typology classification. For residential buildings, the typology classifications were reported at different levels of resolution and with varying terminology. Studies using statistical data sets at the scale of building stocks classified the buildings as "residential buildings" or "dwellings" without further differentiation $[9,19,71]$. The other studies mainly differentiated between sub-typologies "multi-residential" (most studied, including social housing), and "single-family houses" (attached and detached), combinations of both or specific typologies such as "student housing" [5]. For multi-residential buildings and social housing, studies varied in their spatial granularity with equal shares between apartments and the overall building, with one study investigating individual rooms [72]. The difference in spatial granularity is likely to limit the comparability of results, especially among residential buildings. Single-family buildings were investigated at building scale. Apart from the country scale data sets, the number of investigated entities for multi-residential buildings was largely below 10 , with fewer studies in the range between 11 and 100 and a small number of studies above $100[73,74]$. For single-family houses, the number of investigated buildings was equally distributed in the range between 1 and 10 as well as 11 and 100, with few studies above $100[75,76]$. The only other building-related information was dwelling size, reported by few studies $[17,77]$. It can be concluded that the resolution of available information on the investigated buildings tends to be low. The terminology around the residential typologies can be ambiguous and the scale of investigation varies from whole buildings to single apartments and rooms. Moreover, the apartment size, which would have a significant impact on heating and cooling energy consumption, is largely not reported, with the exception of single studies from China [7], Iran [13], Kuwait [59], and Saudi Arabia [20].

The construction year of the buildings is relevant to the applicable building directive or building code. Approximately $48 \%$ of the reviewed studies recorded relevant information about the building construction year and other timelines relating to renovations and retrofitting. In Figure 5, the studies are organized based on the construction year and country. Approximately $76 \%$ of the studies were conducted on buildings constructed or retrofitted after 2006, followed by the studies conducted on buildings built between 1971-1980 and 1946-1970 (with approximately 8\% each). However, it should be pointed out that this statistic is not indicative of the number of buildings considered by the individual studies. In cases where studies span several locations with relevant year of construction data such as in [78], they have been associated with their corresponding countries. 
Distribution by Construction Year Per Country
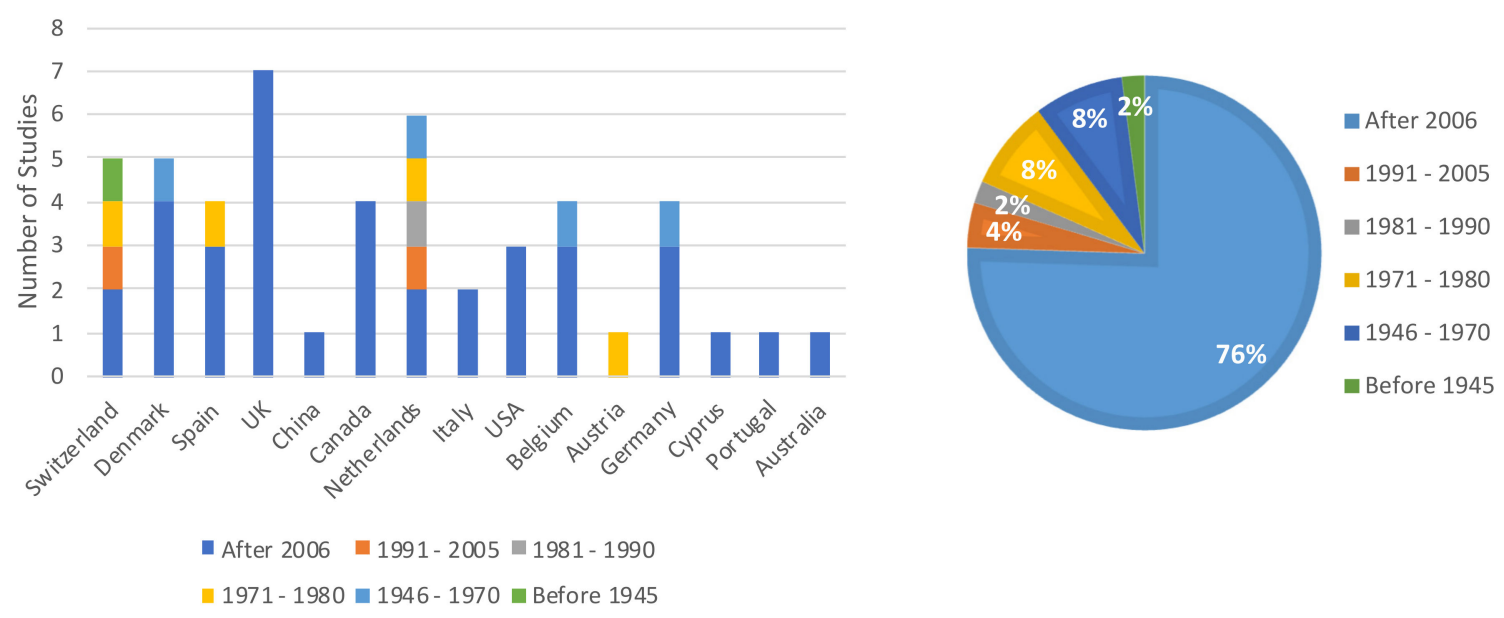

Figure 5. Classification of the studies with regard to construction year and country.

\subsection{Basic Characteristics of Occupants in the Studies}

With regards to occupants, the investigated papers were reviewed from two different perspectives, firstly occupant characteristics and secondly occupant behaviors. For the purpose of this paper, characteristics were defined as socio-demographic information or mindset, which are not consciously changed on a short time scale to adapt to comfort and energy performance. In contrast, behavior relates to active and conscious behaviors and observable actions that reveal patterns over a shorter time frame (i.e., hour, day, season).

Occupant characteristics are generally underreported in the investigated studies, with less than $20 \%$ of all studies reporting any information at all. The reported information is almost exclusively from residential contexts. The characteristics reported are generally inconsistent across the studies due to differences in research foci and data availability issues. The most reported characteristics are the number of people $[9,10,15,26,33,49,59,60,79-81]$, age $[9,10,15,17,21,26,33,59,77,80,81]$, household composition $[6,7,12,14,17,30,59,77,80,82,83]$, and income $[9,10,21,26,29,33,79,80]$. Additional characteristics reported were ownership status $[9,10,26,79,80,84]$ and education levels $[17,21,26,59]$, with sporadic mentioning of physical condition [17], country of origin [73], sex [17,21,59], race [21], and occupation [21]. A spectrum could be observed across the use of generic statistical occupant data at country scale and more individual observations of characteristics derived from a specific building in its cultural and social context. For example, the largest number of different occupant characteristics is reported in studies using country scale statistical data sets for the overall residential building stock $[9,80]$. However, this is due to the nature of the dataset, and does not necessarily mean that these characteristics are the most important ones in the context of the performance gap. In contrast, studies from Kuwait and Saudi Arabia [20,59] reported more in-depth occupant characteristics derived from a sample much smaller than the large-scale statistical data. These reveal important cultural differences in household composition, unit size, and use patterns in comparison to the European studies. The studies which reported occupant characteristics were with few exceptions $[7,20,21,59]$ exclusively from European countries, and thus may not be applicable to other contexts.

It appears that occupant characteristics were not the focus of the investigated papers and thus reported data are limited to available or accessible data. The reviewed papers display a focus on quantifiable characteristics, with little consideration of more qualitative characteristics such as health and cultural background. The currently available information does not allow for the identification of those occupant characteristics that may be important in the context of the performance gap.

Papers were further examined with regard to provided OB-related information, including occupancy patterns and control actions such as changing the heating or cooling 
temperature set-points and operating the windows. A large number of the reviewed papers used behavioral assumptions derived from the standards or provided no details in this regard. In $25 \%$ of the studies, some data on occupant characteristics are included. Most of these studies provided data on the number of occupants, the age of the occupants, household composition, and occupants' employment type. Data on education level, gender, income, ownership and the physical condition of the occupants are also included.

In $44 \%$ of the studies, such data are collected via different means, including surveys $(35 \%)$, interviews $(11 \%)$ and observations $(15 \%)$, sensor-based measurements $(35 \%)$ or via Building Management System (BMS) (2\%). One study [85] gathered OB data through virtual reality. Similar to the occupant characteristics information, the captured behavioral information is also largely focused on residential contexts, social and student housing, and a few instances of university buildings (e.g., $[5,6,9,10,12-14,16,18,49,50,68,77])$. The monitored behavioral information primarily relates to the number of occupants, occupancy schedule, and systems control habits concerning, for instance, operational set-points, the use of appliances and (natural or mechanical) ventilation. Other investigated behavior types include the use of solar shades, blinds, luminaires, and hot water. A few studies include collected data on the activity patterns and clothing behavior. A limited number of the studies conducted surveys and collected some information about the thermostat adjustment frequency, the usage of equipment and appliances, window operation, as well as occupancy patterns. Note that each of the studies captured some but not all of the parameters mentioned above. To consider a level of diversity in occupants' behavior, some studies associated occupants' control habits with behavioral styles in terms of austerity, normal, and wasteful $[64,86]$. In one study, monitored data were used to develop the probability profiles for occupants' presence and control actions in different rooms of the single-family houses under study [10]. This study demonstrated the importance of in-situ measurements and surveys in defining the occupants' interactions with buildings. The review of the $\mathrm{OB}$ reported in the investigated studies highlights the lack of a structured and detailed reporting of the monitored occupancy. Note that the availability of this information is essential if the studies are to provide insights into the role of building occupants in the EPG.

\subsection{Sources of Data}

\subsubsection{Empirical Data}

To be able to assess the performance gap, both empirical data and predicted data are required. The empirical data usually pertain to energy use, user behavior, indoor environment, and outdoor environment.

From 64 papers that reported data on energy-related measurements, parameters such as type of the demand (heating, cooling, ventilation, plug loads, lighting, etc.), measured energy type (electricity, gas, heating demand, etc.), the source of data (bills, metering, etc.), as well as spatial and temporal granularity information were included to some extent. Energy data are not consistently reported. Some cases document final energy (electricity, natural gas, heating oil, etc.), whereas others mention net energy (space heating and cooling loads, domestic hot water). Electricity was primarily used for lighting, plug loads, appliances, and auxiliary equipment of the HVAC system. The most common sources of energy data were bills (electricity, gas consumption) and data from principal meters and submeters. There were very few studies $(7 \%)$ that had dedicated energy use metering $[15,37,66,83,87]$.

Statistical data on building stock were also a source of data for large-scale projects $[62,75,80,88]$. Most of the publications (76\%) used aggregated annual data on energy use, while $10 \%$ of studies used monthly data. The rest of the studies $(14 \%)$ included high-resolution data, involving $5 \mathrm{~min}$ intervals [15,83], $15 \mathrm{~min}$ intervals [38,58,74], $30 \mathrm{~min}$ intervals [35], and daily measurements [22,48,89]. In terms of spatial granularity, studies on non-residential buildings focused on reporting energy use mostly per building and 
rarely per floor [51]. In case of residential buildings, data concerned both building and apartment/dwelling levels, and occasionally room level $[29,44,90]$.

Indoor conditions were monitored in $20 \%$ of the selected studies to evaluate discrepancies between assumed and actual indoor conditions. Air temperature was the most commonly monitored indoor parameter to investigate the performance gap, followed by the relative humidity $[7,10,12,14-16,49,72]$ and the $\mathrm{CO}_{2}$ concentration levels $[10,14-16,27,35,53,54,83,91]$, which were often used as a proxy for occupancy. Indoor environmental conditions were usually monitored via sensors placed in dwellings, but rarely in non-residential buildings. Only two studies monitored operative temperature [83,91] and only one TVOCs [27]. In a third of the studies, the deviation of the actual measured indoor temperatures from the assumed set-points was used to explain the performance gap.

Outdoor conditions are monitored in a number of studies (31\%) in order to normalize the measured energy use data and to examine if the assumptions in the energy simulations regarding the outdoor climate apply $[9,10,12,15-17,21,25-27,31,33,35-$ $37,40,43,45,52,56,57,89,92,93]$. In the other $69 \%$ of the studies, outdoor conditions were not monitored or were not disclosed in the article. In $21 \%$ of the studies, the data on the outdoor conditions were obtained from a (national) climatic institution, hence not directly via on-site measurements $[9,10,21,26,33,94]$. When on site measurements were performed, air temperature data were monitored. Additionally, some studies measured the wind speed [15,27,45], wind direction $[27,45]$, solar radiation $[15,27,31,37,45,56]$, precipitation [15], relative humidity [15,45], as well as VOC's [57]. A few studies did not explicitly mention which variables were measured but maintained that there was a weather station on site $[12,36,50,52,92]$.

\subsubsection{Basis for Predicted Energy Use}

Predicted energy use data are based on standard assessment procedures (SAPs), (dynamic) simulations, or taken from existing databases. SAPs are either based on general assessment tools (e.g., the Passive House Planning Package, PHPP) [77] or national standards, such as the French [19], British [14,23,30,35,93], Belgian [10], Dutch [33,71], Swiss [89,95], Danish [75], Spanish [47], South African [23], or German [15,37,49,56,87] standards. The vast majority of SAPs provide energy estimates in the form of energy use intensities (e.g., in $\mathrm{kWh} \cdot \mathrm{m}^{-2} \cdot \mathrm{a}^{-1}$ ) per building type and characteristics, or, in the case of certification-based standards (e.g., [33]), per certification level. Distinctions are typically made between different energy types (e.g., electricity and natural gas) and end-uses (e.g., heating, hot water, and lighting).

The second source of energy predictions is based on simulations, mainly performed using software tools such as EnergyPlus (e.g., [15,45,96]), TRNSYS (e.g., [94,97]), or IES (e.g., $[8,20])$. These applications offer energy prediction capabilities with high temporal granularity (e.g., per minute) and spatial granularity (e.g., per zone or per room). However, in most reviewed articles, the software applications are used to extract aggregated data to allow benchmarking against monitored data with similar granularities. Common metrics include absolute energy consumption (e.g., in kWh) [16], energy use intensity (e.g., $\mathrm{kWh} \cdot \mathrm{m}^{-2} \cdot \mathrm{a}^{-1}$ ) [17], or a similar $\mathrm{CO}_{2}$-focused metric (e.g., $\mathrm{kg} \mathrm{CO}_{2} \cdot \mathrm{m}^{-2} \cdot \mathrm{a}^{-1}$ ) [8]. Distinctions are often made between different fuel types and end-uses. Some authors focus their analysis on one of these metrics (e.g., heating consumption in [48]), while others target multiple metrics (e.g., space heating, domestic hot water, ventilation, and lighting in [77]). It is important to note that many studies do not provide information regarding occupant related model assumptions or how default values provided by the simulation software are used.

Databases used to derive predicted energy use data are for example the SHAERE database (Sociale Huursector Audit en Evaluatie van Resultaten Energiebesparing) [98], the Kwalitatieve Woning Registratie (KWR) of the Ministry of Housing of the Netherlands (VROM) [9] or the Rekenkamer dataset from Amsterdam [33]. These databases or datasets 
are typically composed of data from energy certificates collected by national municipalities, housing authorities, or other relevant entities.

\subsection{Approaches to Normalization}

Normalization approaches are commonly used to isolate the contribution of occupantrelated factors to the EPG. Hence, the influence of other factors (e.g., weather conditions, construction data) must be accounted for in the calculation method. The aim is to facilitate a valid comparison of the predicted energy demand and the subsequent observed energy consumption of the building. Normalization (or "correction") with regard to weather conditions enables a proper comparison of predicted and actual energy use. It can be combined with other normalization steps regarding, for instance, building geometry, construction and occupancy patterns when comparing before-after energy use or simply differences in the energy use of different buildings. Calculated energy performance indicators (PIs) in directives and standards are commonly expressed in area-related terms $\left(\mathrm{e} . \mathrm{g} ., \mathrm{kWh} \cdot \mathrm{m}^{-2} \cdot \mathrm{a}^{-1}\right)$. In some papers, other expressions of PIs are used for comparison. PIs might be expressed, for instance, in reference to the number of occupants $\left(\mathrm{kWh} \cdot\right.$ person $\left.^{-1}\right)$ [15], to the hours of system operation $\left(\mathrm{kWh} \cdot \mathrm{h}^{-1}\right)$, or to climate-related terms $\left(\mathrm{kWh} \cdot \mathrm{HDD}^{-1}, \mathrm{kWh} \cdot \mathrm{CDD}^{-1}\right)$ [99]. Another instance of normalization in the reviewed literature involved the temperature set-point (e.g., [40]).

Among the different instances of normalization, the one referring to weather is most common. This approach relies mostly on the use of the degree days method [100] and was applied in a considerable number of reviewed papers $[5,10,15,19,33,40,43,62,71,79,80$, $89,93,94,101,102]$. Heating and cooling degree days (HDD/CDD) are a measure of how cold or warm a particular geographic location is during a given period of time. In order to normalize the measured energy consumption for heating or cooling at a site, the $\mathrm{HDD} / \mathrm{CDD}$ values are calculated from the measured weather data (temperature) and are used to modify/adjust the measured energy consumption. These measures are purely temperature-based. As such, they do not consider other potentially relevant climatic influences on buildings' energy performance, such as solar radiation, humidity, and wind speed. In Berggren and Wall [94], the energy use for heating is normalized by using the energy index [103]. This index is defined as the ratio of the measured heating degree days to the standard heating degree days (both adjusted for solar radiation and wind). A number of other studies also considered normalizing the heating energy based on weather or climate, but did not include an explicit specification of the applied method $[26,37,56,58,92]$. Sonderegger [104] normalizes the "variations caused by the "obvious" physical features from the 205 houses" on the energy (gas) consumption. Thereby, the measured gas consumption is compared to the gas consumption as estimated by a regression model [104].

A frequent issue when normalizing energy use for heating is whether or not the DHW is included in the available data. If this is the case, the DHW has to be subtracted from the total heating energy use for a correct comparison. One way to do this is to estimate the DHW heating consumption by averaging the mean daily power of the heating system when the mean ambient temperature exceeds a fixed threshold (e.g., $23^{\circ} \mathrm{C}$ ). Subsequently, the calculated mean power is multiplied by the number of hours to obtain the annual heating demand for DHW. However, as pointed out in IEA SHC Task 44 [105], the downside of this method is that the selected threshold applies only to the summer months. Both summer vacation and the general rise in hot water consumption in winter can lead to a miscalculation of the DHW demand. Due to this circumstance, Mojic et al. [89] increase the DHW heating consumption determined from the power characteristic by a constant rate of $15 \%$.

Some publications normalize energy-related OB in buildings. For example, [94] replace the measured value for DHW with assumed normal use in order to compensate for energy losses included in the measured value. Moreover, the heating energy consumption is reduced by $5 \%$ per each degree Celsius whenever the measured indoor temperature was higher than the assumed value. Following the Swedish recommendations for boundary 
conditions, they assume that only $70 \%$ of lighting and plug loads contribute to internal heat gains. Delghust et al. [40] normalize for temperature set-point, whereas [29] considers temperature set-point and ventilation hours for normalization. In the context of energy consumption for lighting, Motamed et al. [34] consider the impact of different occupancy densities.

To reduce the gap further, another approach besides normalization is to consider net energy (obtained as per standard calculation methods) instead of the final energy. This eliminates influencing parameters including operational faults as well as system efficiency for (heat) generation, storage, and distribution (e.g., sub-metering of flats [15,37,49]).

Among the reviewed papers, a few mention only the variable relevant to normalization and not the normalization method $[9,11,75,95,106]$. Table 2 provides a summary of the most frequently applied methods for normalization and the related normalized variables, together with the respective references. Surprisingly, the large majority $(60 \%)$ of the reviewed publications did not include any information on normalization and are thus not included in Table 2.

Table 2. Summary of the normalization variables and methods.

\begin{tabular}{|c|c|c|}
\hline Normalization Method & Normalized Variable & References \\
\hline Heating degree day (HDD) & Space heating or gas consumption & {$[5,10,15,19,33,40,43,71,79,80,89,93,101,102]$} \\
\hline Cooling degree day (CDD) & Cooling & {$[62]$} \\
\hline $\begin{array}{l}\text { Based on climate/weather } \\
\text { (method not further specified) }\end{array}$ & Space heating & {$[26,37,56,58,92]$} \\
\hline $\begin{array}{l}\text { Measured energy use for heating is: } \\
\text { (i) adjusted by } 5 \% \\
\text { for each degree Celsius deviation from } \\
\text { the average indoor temperature; (ii) } \\
\text { adjusted considering that the share of } \\
\text { internal loads that may affect heating is } \\
\text { fixed to } 70 \% \text {; (iii) normalized using the } \\
\text { energy index (ratio of measured to } \\
\text { assumed HDD). }\end{array}$ & Space heating & {$[94]$} \\
\hline $\begin{array}{l}\text { Elimination of variations by physical } \\
\text { features: measured consumption is } \\
\text { normalized by the amount of energy that } \\
\text { "should" have been used (calculated from } \\
\text { regression model for each house). }\end{array}$ & Gas consumption & [104] \\
\hline Not specified & Space heating & [106] \\
\hline Not specified & Heating and DHW & {$[9,75]$} \\
\hline Not specified & $\begin{array}{l}\text { Final/primary energy use (for heating } \\
\text { and DHW) }\end{array}$ & {$[11,95]$} \\
\hline $\begin{array}{l}\text { Averaging the daily mean power of the } \\
\text { heating system when the mean ambient } \\
\text { temperature exceeds } 23^{\circ} \mathrm{C} \text {. Mean power } \\
\text { was multiplied by the number of hours to } \\
\text { obtain DHW heating demand. }\end{array}$ & DHW & [89] \\
\hline $\begin{array}{l}\text { Replace measured value for hot water } \\
\text { with assumed normal use, excluding } \\
\text { energy losses due to hot water circulation } \\
\text { (compensated as space heating). }\end{array}$ & DHW & [94] \\
\hline Used standard deviations from the mean. & Temperature set-point & [29] \\
\hline $\begin{array}{l}\text { The temperature set-point was estimated } \\
\text { based on the temperature profile during } \\
\text { occupancy. }\end{array}$ & Temperature set-point & [40] \\
\hline $\begin{array}{l}\text { Consider the type of ventilation with } \\
\text { most hours (grills, windows, } \\
\text { mechanical systems). }\end{array}$ & Ventilation hours & [29] \\
\hline
\end{tabular}




\subsection{Magnitudes of Performance Gap}

This section discusses the magnitude of the performance gaps associated with $\mathrm{OB}$ observed in previous studies. Only studies that included monitored data on energy use and occupants, or at least energy use measurements, were included, such that the reported EPG could be classified as evidence-based (i.e., the subset of studies classified as "gold" and "silver" in Section 2.1). As mentioned before, the EPG magnitude is calculated as a deviation of the measured energy use from the expected energy demand at the design stage [87]. The expected or predicted energy demand is typically estimated through standard assessment procedures numeric simulation, or is taken from existing benchmarking databases.

Of all the studies, 68 reported a quantified performance gap and are shown in Figure 6. Studies including only one building are represented by circular markers. For studies that included multiple buildings, and thus multiple EPG magnitudes, the gaps are represented by a range. The EPG across all studies is, on average, $55 \%( \pm 89.8 \%)$. Figure 7 shows mean and median EPG magnitudes separately for residential and non-residential buildings.

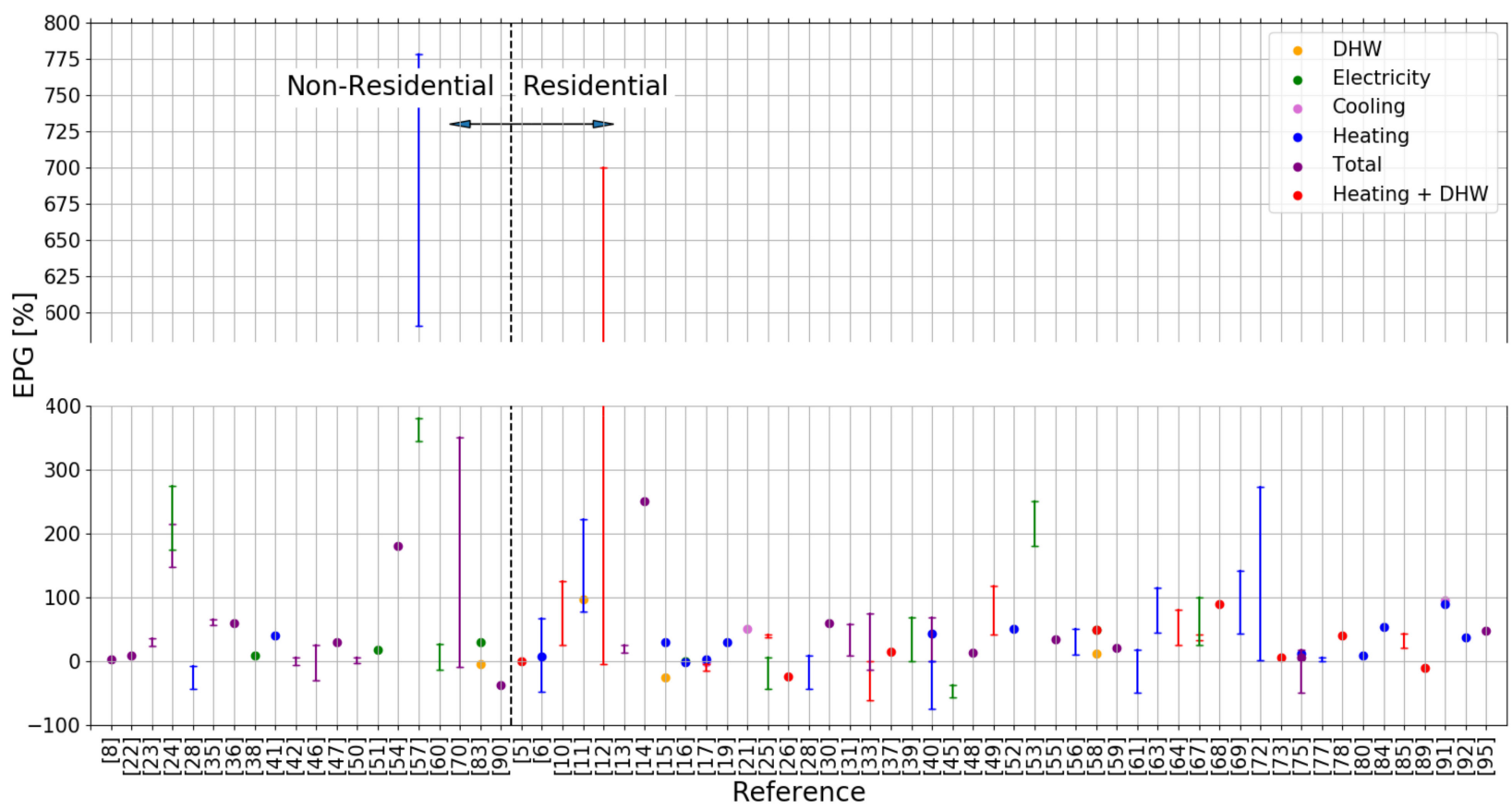

Figure 6. Distribution of the EPG between the residential (43) and non-residential buildings (17).

The reviewed studies did not report the EPG consistently. For example, some studies reported EPG as a percentage or in absolute terms in units related to total energy or area-normalized energy use, while a few reported their results in terms of $\mathrm{CO}_{2}$ emissions (e.g., [8]). Some studies reported gaps with respect to total building energy consumption, while others reported detailed gaps for one energy source (e.g., natural gas or electricity). Another group of studies focused on the energy end use (e.g., domestic hot water, heating, cooling), but this approach was typically used when studies compared the performance of different functional units such as a whole building compared to an apartment or commercial unit (e.g., $[15,37,49])$. 


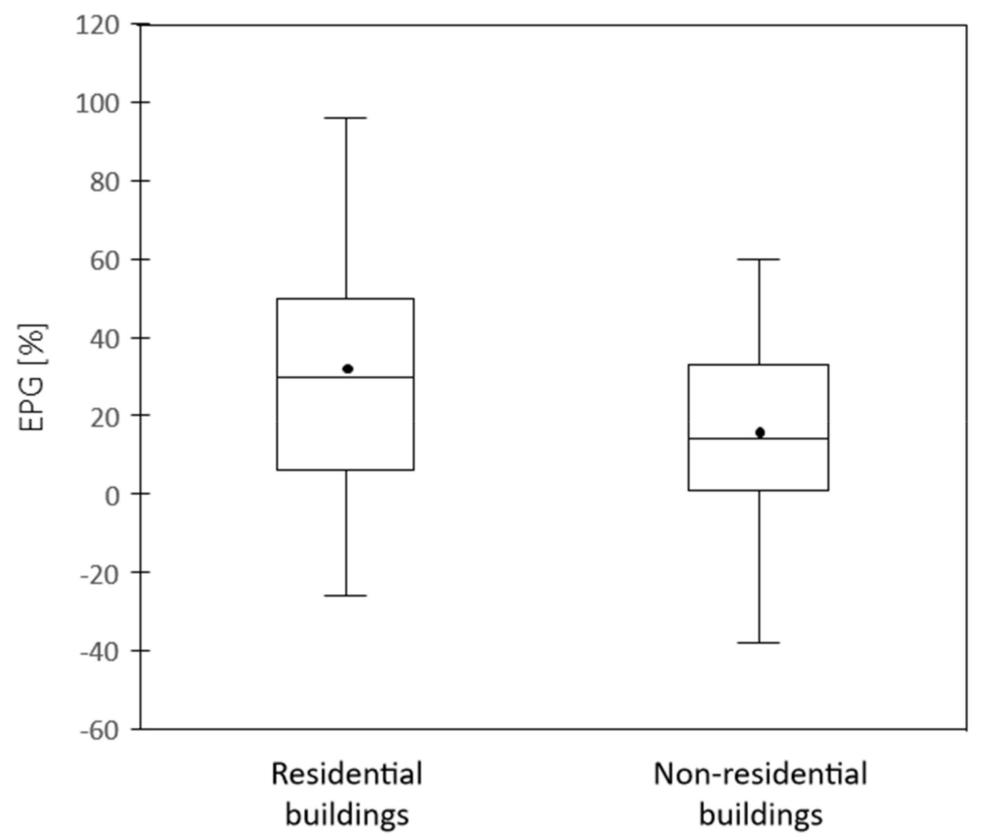

Figure 7. Distribution of EPG (in \%) for residential and non-residential buildings.

Note that the heterogenous nature of the approaches of the reviewed studies (object specification, data collection, data resolution, estimation methods, normalization procedures) as well as certain levels of existing opacity and inconsistency in the reporting of the results make it difficult to formulate general findings regarding the magnitude of the EPG. To illustrate this challenge, consider a listing of exploratory inquiries that could be processed via a meta-analysis of a set of consistently structured and reported studies. Such a listing could include, for instance, the following conjectures:

(i) Given the assumption that occupants, in residential buildings, tend to have more control over systems and envelope operation, it is less likely for predictive models to capture the dynamics and variance of occupants' behavior, resulting in a potentially larger EPG.

(ii) Energy usage dependent on $\mathrm{OB}$ (e.g., lighting) is more difficult to predict and may thus result in larger EPG magnitudes as compared to energy usage that is less or not at all dependent on occupant intervention (e.g., continuously operated ventilation system).

(iii) The expression of the EPG in relative terms (i.e., in percentage) is likely to be larger in the case of highly energy-efficient buildings, as even relatively small differences between modelled and actual values can result in high relative EPG magnitudes.

(iv) The application of detailed numeric energy simulation methods would yield smaller EPG magnitudes as compared to energy estimates generated by default values based on standards. Likewise, calibrated energy models of existing buildings could be expected to result in smaller post-retrofit EPG.

The information provided in the reviewed studies could only contribute to the clarification of the first conjecture above. As shown in Figure 7, the median gap is larger in residential buildings ( $30 \% \pm 51 \%$ ) than in non-residential buildings (14\% $\pm 27 \%)$. Likewise, standard deviation is larger in the former case. This may be explained in part by larger differences between assumed and measured temperatures in several residential studies. However, the smaller sample size of the non-residential buildings may have also been responsible for the reported larger EPG magnitude in the case of residential buildings. 
Due to the aforementioned inconsistency between studies in the presentation of findings, it was not possible to judge the validity of the second conjecture in the above listing (dependency of the EPG magnitude on the level of occupants' control). As the reported gaps regarding electricity usage were not separated by energy-end use, the gap size between occupant-controlled and non-occupant-controlled loads could not be determined. Likewise, an examination of the third conjecture (higher sensitivity of energyefficient buildings to occupant-driven loads) was not possible due to insufficient data availability. Construction dates were generally reported; however, whether a building was standard or high-performance was not reported consistently. Finally, the examination of the last conjecture (lower EPG magnitudes in cases involving the deployment of detailed simulation) was hampered due to insufficient evidence (e.g., [15,48]).

\subsection{Identified/Assumed Causes of Performance Gap}

As described before, the selected studies were reviewed to examine suggested causes of the performance gap. Thereby, we first classified the studies according to their objectives and context (Section 3.7.1) followed by a discussion of the causes of the EPG related to occupants (Section 3.7.2), the drivers of occupants' behavior (Section 3.7.3), and, lastly, other contributors to the EPG (Section 3.7.4).

\subsubsection{Approaches to Quantification of Gap}

The reviewed papers were first classified based on their objectives into three groups: (a) those that consider multiple performance gap causes (both occupant and non-occupant related); (b) those focusing on occupant-related causes; and (c) those with other objectives or foci. Out of these three, group (b), which focused only on the occupant-related causes, included the least number of papers. Next, the causes were grouped according to their relevance to the occupants. Occupant-related causes include occupants' presence and behavior. Other causes of the performance gap are those related to buildings' design, construction, and operation. Both categories are explained in detail in Section 3.7.2, Section 3.7.3, and Section 3.7.4.

The aforementioned gold, silver, bronze classifications from Section 2.1 identify which studies contained energy data, occupant data or both. However, a further classification was required regarding the context of the study in terms of the methodology employed and the type of evidence presented to support the identification of the gap cause. Hence, the studies were further categorized as follows (see Figure 8):

- Experimentally-based studies: Comparisons were made between data collected from the same building at different times or concurrently from very similar buildings or units. For instance, energy consumption in various buildings with identical attributes (type, geometry, construction and systems, climate) was compared to determine the reason for the observed differences in energy performance.

- Modeling-based studies: Comparisons were made between various cases exclusively via simulation. For example, a computational study that explores the impact of two different occupancy schedules on energy consumption falls into this category.

- Combined modeling and experimental studies: Comparisons were made between data collected from the building and those obtained from the computational (simulation) model of the building. For example, actual energy consumption in a code-compliant building was compared to the building's energy model.

- Other studies: These studies included discussions of performance gap causes elicited from other sources such as expert opinion, surveys, review of other studies, etc. 


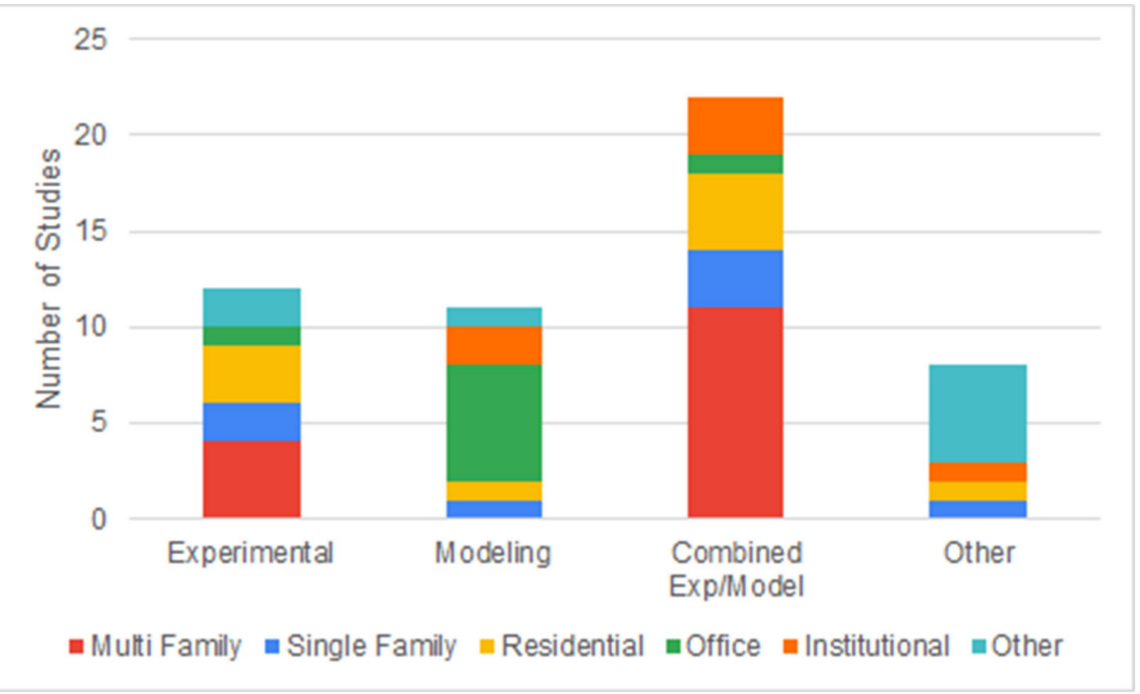

Figure 8. Distribution of studies' methods broken down by building type.

The most common approach to assessing the performance gap is to compare actual building data with modeled data ("Combined Exp/Model" in Figure 8). This appears plausible given that many performance gap investigations are undertaken to determine why a building is not performing as the design-stage prediction suggested. Additionally, this combined approach allows for easier normalization of factors beyond the researchers control, such as weather or occupancy status. However, one drawback of this approach is the potentially unrealistic assumptions about building operation during the prediction phase, if these assumptions are not updated according to the actual building operation.

The review of the reported causes of the performance gap revealed that whereas in some cases such causes are directly based on evidence, in other cases they are simply presumed. We refer to these as "Actual" and "Assumed". "Actual" causes in an experimental context are relevant to instances where the researcher used collected data in the field to identify the performance gap cause. For example, it was suggested that a difference between monitored set-point temperature and the initially assumed set-point was correlated with a deviation of the metered energy use for space conditioning from the expected value. In a modeling context, "Actual" causes included instances where the researcher varied parameters in a model to demonstrate how different aspects of building function would impact performance. This involved, for example, modeling the differences in occupancy schedule and predicting the impact on lighting energy use. "Assumed" causes were instances where the researcher made assumptions about the performance gap cause based on anecdotal observations that were not supported by collected detailed data. For example, occupants' statements regarding daily behavioral practices were found to be consistent with the performance gap as manifested in energy bills. For a subset of 46 studies for which the cause determination was examined, $56 \%$ were considered to have identified actual causes and $44 \%$ had assumed causes.

\subsubsection{Occupant-Related Contributors to EPG}

The review revealed that the majority of the reviewed papers (more than $70 \%$ ) report a form of occupant-related cause for the performance gap-either identified or presumed. These studies are summarized in Table 3 and discussed in the remainder of the section. The occupant-related contributors to the performance gap were grouped into four categories, according to the building model component they influence. These categories are envelope, mechanical systems, plug-loads and lighting, and internal heat gains. 
Table 3. Overview of occupant-related EPG contributors in different categories.

\begin{tabular}{|c|c|c|c|}
\hline Category & Building Model Ingredient & $\begin{array}{l}\text { Occupant-Related Performance } \\
\text { Gap Contributors }\end{array}$ & References \\
\hline Envelope & $\begin{array}{l}\text { Operation schedules of windows } \\
\text { and shading devices (e.g., blinds) }\end{array}$ & $\begin{array}{l}\text { Occupants opened windows more } \\
\text { frequently or for longer periods } \\
\text { (as compared to model } \\
\text { assumptions) } \\
\text { Occupants turned off the installed } \\
\text { MVHR (mechanical ventilation } \\
\text { with heat recovery) and used } \\
\text { windows instead for ventilation } \\
\text { Discrepancies between assumed } \\
\text { and actual operation of shading } \\
\text { devices resulting in the deviation } \\
\text { of actual solar gains from model } \\
\text { assumptions }\end{array}$ & $\begin{array}{c}{[5,6,12,14,18,22,28,32,37,45,52,} \\
54,55,58,67,72,77,79,82,89,95]\end{array}$ \\
\hline \multirow{3}{*}{ Mechanical systems } & \multirow{3}{*}{$\begin{array}{l}\text { Set-point temperature, thermostat } \\
\text { settings, system operating } \\
\text { schedules and settings }\end{array}$} & $\begin{array}{l}\text { Higher actual indoor } \\
\text { temperatures than those assumed } \\
\text { in the model }\end{array}$ & $\begin{array}{c}{[6,9,11,14,15,18,29,37,53,55,58,} \\
75,79,82,90,101,104,107,108]\end{array}$ \\
\hline & & $\begin{array}{l}\text { Lower indoor temperatures or } \\
\text { shorter heating durations than } \\
\text { assumed }\end{array}$ & {$[19,33,40,45,109]$} \\
\hline & & $\begin{array}{l}\text { Schedules of the ventilation } \\
\text { system and air flow rates do not } \\
\text { match actual occupancy patterns }\end{array}$ & {$[10,75,95]$} \\
\hline $\begin{array}{l}\text { Plug-loads and } \\
\text { lighting }\end{array}$ & $\begin{array}{l}\text { Occupant density and/or } \\
\text { schedule }\end{array}$ & $\begin{array}{l}\text { Discrepancies between actual and } \\
\text { assumed occupant density or } \\
\text { schedule lead to higher or lower } \\
\text { use of IT equipment, lighting and } \\
\text { appliances } \\
\text { Use of secondary heating/cooling, } \\
\text { such as electric heaters }\end{array}$ & $\begin{array}{l}{[7,16,21-23,30,35,36,38,39,41,42,} \\
47,50,51,57,59,60,65,67,110,111]\end{array}$ \\
\hline Internal heat load & $\begin{array}{l}\text { Occupant density and/or } \\
\text { schedule }\end{array}$ & $\begin{array}{l}\text { Standard occupancy schedules } \\
\text { imply high heat gains, which can } \\
\text { result in underestimation/ } \\
\text { overestimation of energy use for } \\
\text { heating/cooling }\end{array}$ & {$[16,17,75]$} \\
\hline
\end{tabular}

The envelope category mainly entails operation schedules of windows and shading devices (e.g., blinds). The frequency of window opening is one of the most recurrent occupant-related candidate causes of the EPG, appearing in 36\% of the studies listed in Table 3. For instance, actual heating demand was found to be higher than expected, as occupants opened windows more frequently or kept them open longer than assumed. Other studies $[6,14,49]$ report that occupants turned off the installed MVHR (mechanical ventilation with heat recovery) and used windows to ventilate instead, with significant energy implications. Similar discrepancies were found with the operation of shading devices $[6,22,23,88,90]$ in approximately $10 \%$ of the above studies, leading to higher or lower solar gains than modelled.

Relevant to the "mechanical systems" category are set-point temperature, thermostat overrides, system operating schedules, and settings. One-third of the studies in Table 3 report higher actual indoor temperatures than assumed $[6,9-11,14,15,18,29,37,53,55,58,75,82$, $91,101,104,107,108]$. This discrepancy was seen in some instances as the factor responsible for the performance gap [104]. Cuerda et al. [45] noted that the actual heating periods were shorter than those suggested by standard schedules, leading to a lower energy consumption level than modeled. Similarly, there are a few studies (approx. 10\%) that report lower indoor temperatures or shorter heating durations than assumed. In some studies $[10,75,95]$, 
a discrepancy was found between the schedules of the ventilation system and air flow rates on the one hand and the building occupancy on the other hand.

The plug-loads and lighting category pertains mainly to assumptions regarding occupant density and/or schedule. Discrepancies between actual and assumed occupant density or schedule can explain higher or lower use of IT equipment, lighting, and appliances as compared to respective expectations, as seen in nearly $40 \%$ of the above studies. Four studies $[41,47,51,67]$ report that office equipment and lighting that remained switched on outside operating hours resulted in increased electricity consumption. Other plug-load related contributors to the EPG may include the use of secondary devices for heating and cooling, such as electric heaters [95].

The internal heat load category entails occupant density and/or schedule. Occupant density and presence/appliance schedules not only influence plug-loads, but can also lead to discrepancies between assumed and actual internal heat loads. Carpino et al. [16,75] report a case where standard occupancy schedules led to an overestimation of internal heat gains. This, in turn, resulted in an underestimation of the energy use for heating. In another study [17], actual occupancy schedules were found to deviate from standard profiles. This resulted in higher internal heat gains than predicted and, consequently, in a lower heating load and a higher cooling load than simulated. As shown in Table 3, the most frequently identified occupant-related causes in the reviewed literature are plug-load schedules $(40 \%)$, window operation (36\%), and set-point temperature (33\%).

Note that a number of studies among the reviewed articles conduct parametric and sensitivity analyses. Thereby, the occupant-related parameters in a building model are varied to computationally explore their impact on energy performance $[2,69,75,97,112]$. As such, these studies cannot identify or confirm occupants' role in the EPG, but rather estimate the magnitude of their influence under assumed scenarios of $\mathrm{OB}$ variation. These studies are therefore not considered as providing hard evidence for the occupants' role in the performance gap.

\subsubsection{Drivers of Occupant Behavior Leading to EPG}

We discussed above the assumptions regarding occupant presence and behavior in different categories and how they can influence the estimated or modeled energy consumption and thus contribute to the EPG. A further level of analysis involves the exploration of the background of the behavior itself, which may be related to occupants' socio-economic characteristics [113]. This background includes, for instance, income [19,76,80,114], lifestyle (e.g., employment status) [17,30], energy billing practice [12], environmental attitude [27,115], occupant expectations [18], building's energy efficiency level [29], and renovation versus new construction [80]. In certain cases, the improper operation of systems (contrary to their intended use as designed/simulated) may be the consequence of the inadequate design of control interfaces $[6,14,53,116]$.

In the majority of the reviewed articles, the underlying cause of the reported $\mathrm{OB}$, assumed to be responsible for the EPG, was not explored. When the rebound effect is directly addressed, it is attributed to psychological mechanisms, e.g., lifestyle changes, moral licensing [13], and lack of knowledge [49]. The prebound effect is seen mainly resulting from low income and fuel poverty $[76,109]$.

\subsubsection{Other Potential Contributors to EPG}

Besides occupant-related factors, the reviewed studies also considered other drivers of the EPG. These can be classified as related to building design, construction, and operation. During the design phase, poor, overly simplified, or unrealistic modelling assumptions can lead to the overestimation or underestimation of the predicted energy use of buildings. Improper modeling assumptions can pertain to, for instance, buildings' space usage and operational conditions $[13,95,117]$. Similarly, making the right assumptions concerning future projections of contextual factors and boundary conditions such as weather [17] and solar gains [5] remains a major challenge and can be the source of large discrepancies 
between predictions and reality $[50,95]$. Moreover, calculation methods such as those embedded in energy certification tools can also involve technical inaccuracies [76] or inappropriate simplifications [19,47].

The performance gap may also emerge from the building construction process and the resulting frequent discrepancies between the as-designed and the as-built versions of the building. Construction-related contributors to the energy gap can also include the constructed building's deviation from inaccurate model assumptions concerning the building envelope's thermal transmittance $[17,53]$ and air-tightness $[5,17,32]$. Faults in the installation of energy systems represent a common cause of underperformance in buildings [49,117], which could also be due to a lack of proper commissioning [14].

Finally, operation-related issues may also act as drivers of the EPG. The most frequent instances of inefficiencies pertain to the facility management $[14,49,66,95,110]$ or sensor errors and related negative consequences for systems controls [95].

\section{Discussion}

\subsection{Overview}

We discuss in this section the findings of the study in terms of a number of questions raised in the introduction. Specifically, we reflect on the general understanding of the EPG in the literature, we discuss the degree of the representativeness of the reviewed studies, the consistency and quality of required modeling, monitoring, and normalization steps, we look into the evidence for the existence and extent of the occupant-induced EPG, we explore the suggested causes of the occupant-related EPG, and we consider the implications of the findings for future efforts.

\subsection{Views on $E P G$}

As stated at the outset, the primary objective of the present paper is to gauge the existence and extent of evidence for the purported occupant-induced gap between expected (i.e., estimated, calculated, computed, predicted) and actual building-related energy use. As such, the definition of the EPG is not consistent across the board. Implicit definitions of the EPG and their variance in different papers are reflected in the classification of the deployed methods to measure the EPG (see Section 3.7.1). Nonetheless, one major category in this classification (combined use of measurement and modeling) does indeed involve the comparison of the predicted energy use (based on standard calculations or simulations) with the actually monitored energy use (Figure 8 ). However, there are studies that include modeling, but not measurements. Furthermore, there are studies that include monitored energy use, but involve no modeling. The former, purely modeling-based category may provide a sensitivity analysis with regard to the model's response to variations of occupantrelated input assumptions. As mentioned previously, this-useful as it may be for certain considerations-does not yield any kind of hard evidence for the actual relevance of occupants' role in the EPG. The latter, purely measurement-based category approaches the EPG via comparison of the actual energy use of the same building at different times, or through comparison of the monitored energy use of very similar buildings. These types of studies enable the identification of the magnitude of occupant behavior on the energy use. However, without further analysis, they do not identify causes of the EPG. Note that the decisive factor in any EPG analysis, namely the actual energy use, is not reported consistently in the reviewed studies. This especially concerns the resolution of the monitored energy data in view of its spatial and temporal granularity. For instance, only $10 \%$ of studies report monthly energy use data. Some $14 \%$ report higher-resolution data. The rest are based on annual energy consumption values.

\subsection{Building Locations and Types}

The distribution of papers displays a number of limitations both in view of the covered locations and the studied buildings (see Section 3.2) (Figure 4). The majority of the studies $(78 \%)$ were conducted in temperate climates (mostly in Europe). Moreover, a large fraction 
of the studies investigated residential buildings (60\%). Other typologies investigated included offices $(15 \%)$, educational buildings (13\%), laboratories $(1.5 \%)$, and others $(10.5 \%)$. As such, whatever conclusions are derived from the bulk of existing publications on the subject, they cannot be suggested to represent the circumstances globally.

It is hypothesized that it is difficult for researchers to obtain detailed building-related data. Likely sources for larger data sets are governments or housing associations. These could be more indicative of average building stock characteristics rather than the embedded variability. There is a need to identify which building-related parameters are important and must be considered in future studies as well as what should be the proper scale. Similarly, the process of the sharing of building-related data needs to be more efficiently organized.

\subsection{The Role of Occupants}

Generally speaking, some 70\% of the reviewed papers report occupant-related causes of the EPG. However, the strength of the provided evidence varies significantly across the reviewed articles. Around $40 \%$ of the reviewed articles involved empirical data on both energy and occupants. Among these, only about one-third included sensor-based monitoring of the occupancy, and only $2 \%$ included data from BMS (Building Management System). Another $15 \%$ of the papers relied on snap-shot types of observations (e.g., of the state of thermostats). The remainder of the reviewed papers entailed less certain information on occupants, such as surveys (35\%) and interviews $(11 \%)$.

These observations imply that, among all reviewed studies, only $14 \%$ included quantitative data on both energy use and occupant behavior. For this group of studies, the magnitude of the reported EPG can vary significantly. The reliability of the latter inference is of course dependent on the quality of the deployed normalization procedures.

Moreover, the reviewed studies mainly originated from Europe. This means that the global diversity, especially related to different ways of occupying residential buildings in different climate zones or cultural contexts (e.g., family size), as well as different energy prediction in countries are unlikely to be reflected in the investigated papers. More interdisciplinary research using dedicated frameworks or approaches would be helpful to quantify these aspects in the context of the performance gap.

\subsection{Modeling Approaches}

Methods for the estimation of future energy use vary considerably across the studies. Some studies rely on rather simple standard-based calculations (34\%), whereas others deploy simulation tools ( $43 \%$ ). More critically, the majority of the reviewed studies do not provide information concerning the source of occupancy-related model input assumptions. Such circumstances make it difficult to compare and generalize the studies' conclusions regarding the existence and extent of the EPG and the suggested role of occupants therein.

\subsection{Challenges of Normalization}

As alluded to before, in most EPG investigations, the types of modelled and metered energy data are not directly comparable. For instance, whereas the measured energy data may be related to end energy use as inferred from energy bills, the simulation may have been focused on energy loads. Hence, to make meaningful comparisons of modelled and monitored energy data, normalization procedures must be followed. Only $7 \%$ of the reviewed articles could rely on the dedicated monitoring of energy. This implies that, in the overwhelming majority of the existing studies, a direct comparison of simulationbased and monitoring-based space-level energy loads is not possible. This underlines the critical importance of the robustness of the normalization approaches. For instance, a comparison of modelled and actual energy use at the space level would require the isolation of thermal energy delivered to the space. In the absence of a dedicated space-level energy monitoring, measured indoor air temperatures could support the estimation of the respective magnitudes. However, indoor temperatures were measured in only $20 \%$ of the reviewed studies. More importantly, $60 \%$ of the reviewed articles did not include 
any information about normalization. As far as normalization with regard to weather conditions is concerned, the reviewed studies display a number of issues. The reliability of weather normalization in $76 \%$ of the studies is arguably uncertain, as they did not record outdoor conditions. In $10 \%$ of the studies, outdoor conditions were obtained from an existing weather station. Moreover, micro-climatically relevant variables (e.g., temperature, solar radiation) considered for normalization are not consistent across the different studies. Most studies mostly use the aggregate climatic indicator HDD for normalization purposes. This indicator considers only air temperature. Hence, other factors of climate are ignored in the normalization. This suggests that the same EPG investigation could yield different outcomes if researchers would use different criteria and methods for normalization.

Speaking in more general terms, the OB normalization (or more specifically, energyrelated $\mathrm{OB}$ normalization) must be handled with caution; the normalization for the deviation from user behavior or the expected use of the building, such as window opening, shading or indoor temperature, inevitably has an impact on one of the "potential" sources of the performance gap. For example, the normalization of the energy consumption for indoor temperature set-points reduces the effect of the related $\mathrm{OB}$ action (thermostat setting). The theoretical optimum (eliminated gap) would be to normalize the complete user behavior with measurements to calibrate the measured consumption to the calculated demand.

\subsection{The EPG Magnitude}

The EPG magnitude, emerging from the studies, ranges from $-38 \%$ to $+96 \%$ in Figure 7. The mean and median of EPG magnitudes (in percentage) are +37 and +30 for residential buildings and +16 and +14 for non-residential buildings. This would indicate that it is more likely that buildings' energy use is underestimated rather than overestimated. However, this cannot be asserted with certainty, given the previously mentioned unbalanced distribution of the studies (in terms of location and building type).

\subsection{Proposed Measures to Reduce Occupant-Related EPG}

An obvious response to the problem of energy use prediction is the improvement of the prediction models in general and the enhancement of occupancy-related model input assumptions in particular. To this end, studies underline the importance of post-occupancy investigations of buildings' use patterns and client requirements. This rapid feedback loop is assumed to enable building planners and modeling experts to continuously improve the quality of their assumptions regarding building occupants. A key input assumption pertains to the assumed number of occupants and the duration of their presence in the buildings. Likewise, it is essential to ensure that the occupancy profiles adopted by compliance tools are appropriate for the building type. The fidelity and empirical grounding of the occupancy-related model assumptions have been argued to be more essential than the specific algorithmic features of the prediction tools $[118,119]$. As such, even relatively simple calculation methods could yield reasonable results, if they are based on reliable empirical data. Consequently, the use of historical data and the availability of more comprehensive repositories of actual high-granularity occupancy information (covering multiple climatic boundary conditions, building types, populations) could contribute to the improved marksmanship in the representation of occupants in building energy models.

A further, highly important issue pertains to the socially and demographically relevant background of the buildings' occupants. Factors such as family size, income levels, and fraction of energy-related expenditures are suggested to be relevant to occupants' behavior. Such information is rarely considered in the course of energy use prediction processes. Less than $20 \%$ of the reviewed papers included any detailed information concerning the background (household composition, family size, income, age, etc.) of the users of the buildings studied. As such, the judicious use of socio-economic variables in addition to the default technical analysis can contribute to a more realistic assessment of energy use behavior. Model calibration based on actual energy usage is suggested as a further 
remedy. However, strictly speaking, this option applies only to building retrofit scenarios or building operation cases.

Certain recurrent recommendations in the reviewed studies with regard to occupant behavior are worth mentioning, assuming occupants' influence on buildings' energy performance, independent of its magnitude, should be a matter of concern. For instance, it is suggested that there is a need for better information for occupants as to how the buildings' systems and equipment should be properly used. However, recommendations occasionally entail certain contradictions. Whereas occupants' lack of understanding of control systems is mentioned, the authors also highlight the need for occupant-centric buildings and system designs, whose mode of operation could be understood without long explanations. Moreover, buildings' control systems and devices, their interfaces, and their operation regimes could proactively consider and address certain aspects of human behavior. These include, for example, presence detection technologies and smart scheduling procedures. The intelligent automated control of windows, blinds, and luminaires guided, for instance, by monitored levels of $\mathrm{CO}_{2}$ concentration, indoor illuminance, or incident irradiance has the potential to anticipate and accommodate occupants' needs and reduce the probability of counterproductive user actions. Needless to say, efforts could be made to encourage more energy-conscious user behavior, for instance, via information campaigns or dynamic energy-centric feedback mechanisms.

\section{Conclusions}

A key motivation behind the present paper was critical concerns with a relatively recent common narrative in the community of building-related energy efficiency stakeholders. This narrative unfolds along the following lines: our projections of buildings' energy use frequently deviate from their actual energy performance-a circumstance referred to as the EPG. As buildings are increasingly endowed with thermally enhanced envelopes and systems, the relative role of occupants (specifically their energy-relevant behavior) is suggested to have increased, thus becoming the main contributor to this discrepancy. Based on this assertion, a number of inferences are made, two of which pontificate the need for a) more detailed (preferably stochastic) occupant models in energy simulation tools, and b) feedback systems and information campaigns to correct adverse occupant behavior. Notwithstanding the potential and usefulness of these recommendations, the question remains if their underlying premise, namely the assumed centrality of occupants role in the EPG, is sufficiently documented. We pursued this question in terms of the null-hypothesis stated in the introduction of the paper as follows:

There is no conclusive and sufficient empirical evidence supporting the claim that occupants' behavior is responsible for the bulk of building-related EPGs.

In an effort to reject this null-hypothesis, we examined in this paper recent publications relevant to the subject. The focus was mainly on EPG studies concerning the discrepancy between computationally predicted and actual energy use. However, a number of studies were also included that addressed the EPG by the comparison of similar buildings with different occupancy patterns. Furthermore, the selected studies also included a number of cases involving only (typically parametric) simulation.

Notwithstanding the exact definitions, the studies do report a considerable range of the EPG (somewhere between $-38 \%$ and $+96 \%$ ). However, the nexus to the occupants' role is not thereby convincingly established. As summarized in the discussion section, the investigation of the previous research in this area does not provide a basis strong enough to reject the above null-hypothesis. On the one hand, there is a considerable level of inconsistency among the studies in view of the scope of the cases, adopted approaches, the comprehensiveness and quality of collected data, the quality of the normalization procedures (in applicable studies), and the robustness of the conclusions. The inconsistency is reflected in the choice of prediction tools (anything from standard-based simplified calculations to dynamic simulation), spatial (zone, room, apartment, whole building) and temporal (minute, hour, day, month, year) granularity of collected data, real occupancy information 
(none at all, snapshot observations, surveys and interviews, sensor-based monitoring), and factors involved in normalization (energy use versus energy load, construction and systems, indoor and outdoor climate). This makes the potential for meta-analyses-and ultimately generalization - of the reported findings infeasible. Only $40 \%$ of the reviewed publications included, at least formally, what could be considered to constitute the minimum criteria toward an evidence-based confirmation of the purported decisiveness of the occupants' role in the EPG: such criteria would include traceable documentation of the energy prediction models (including, especially, details of occupant-related modeling input assumptions), carefully conducted and transparent normalization procedures, and-most importantly-observation-based documentation of occupants' actual behavior. This percentage further decreases down to $14 \%$ if we look for detailed (sensor-based) monitoring data concerning occupants' actual behavior.

The above observations also constitute the basis for recommendations toward future EPG studies. As such, the community would benefit from consistent standards of research design, such that individual investigations could be synthesized at a higher, more inclusive and representative manner via, for instance, cross-section studies and meta-analyses. To this end, we could reiterate the key general recommendations for improving the quality of future occupant-related EPG studies. Such investigations should:

- Document in a detailed and explicit manner the research design, target buildings, energy use monitoring, and occupant behavior observations.

- Examine the integrity of the energy use prediction tool, its consistent and correct application, and the correspondence of the temporal and spatial resolution of the modeling results with the corresponding monitored energy use data.

- Clearly distinguish between predicted and observed attributes and magnitudes of the energy data (e.g., differentiation between energy loads versus end energy use, as well as differentiation between energy quantities used for separate purposes such as heating, cooling, lighting, and equipment).

- Openly present any kinds of assumptions made to match the granularity of observed and calculated data, for instance, when wholesale energy use data (e.g., annual or monthly energy bills) are computationally disaggregated into subcategories (e.g., cooling versus heating versus lighting).

- Apply systematic and transparent normalization procedures that isolate and eliminate EPG sources not related to occupants' presence and behavior (e.g., the deviation of as-is versus as-planned construction properties and building systems specifications, prevailing external boundary conditions and their deviation from those assumed in the modeling phase).

Needless to say, the failure to reject the above hypothesis does not mean occupants do not have a role in the EPG. Rather, what the results advise against are across-the-board and nonchalant claims about the central role of occupants in the EPG, which are sometimes stated at the outset of otherwise meaningful efforts and contributions toward improved energy efficiency of the built environment. Such meaningful efforts encourage, for instance, the provision of:

(i) Environmental control systems with high zonal granularity.

(ii) Individual control opportunities versus purely central control.

(iii) Smart control features that modulate indoor climate services (heating, cooling, ventilation) depending on the occupant control behavior.

(iv) The pervasive application of occupants' presence information toward energy-efficient system operation in non-occupancy periods.

(v) Adequately designed building control systems with intuitive, transparent, and responsive user interfaces for operation support.

(vi) Effective information (both real-time and historical) to the individual occupants regarding the energy implications of their behavioral patterns.

(vii) Clear and intelligible information regarding the proper operation of control systems and devices. 
(viii) General initiatives and campaigns to raise occupants' level of consciousness, both regarding environmental issues in general and possibilities (such as adaptive behavior) to save energy without compromising comfort in particular.

Most of these efforts represent rationally arguable and common-sense options. As such, their pursuit is entirely justified, and their realization potential would be perhaps even larger if our discourse does not assign the occupants a priori as the main culprits responsible for the EPG, but as partners in a collective endeavor to enhance the energy performance of the built environment.

Author Contributions: CConceptualization, A.M. and C.B.; methodology, A.M., C.B., H.A.; writingoriginal draft preparation, A.M., C.B., H.A., E.A. (Eleni Ampatzi), R.K.A., E.A. (Elie Azar), V.M.B., M.F., J.H., D.K., H.N.K., A.L.-N., A.R., F.C.S., M.S., M.T. (Mahnameh Taheri), D.T., M.T. (Marianne Touchie), S.V.; writing—review and editing, A.M., C.B., H.A., E.A. (Eleni Ampatzi), R.K.A., E.A. (Elie Azar), V.M.B., M.F., J.H., D.K., H.N.K., A.L.-N., A.R., F.C.S., M.S., M.T. (Mahnameh Taheri), D.T., M.T. (Marianne Touchie), S.V.; project administration, A.M., C.B., H.A. All authors have read and agreed to the published version of the manuscript.

Funding: Jakob Hahn gratefully acknowledges the financial support provided by the German Federal Ministry for Economic Affairs and Energy (BMWi), promotional reference 03ET1648A (EnOB: NuData Campus). Matteo Favero would like to thank the Research Centre on Zero Emission Neighborhoods in Smart Cities (FME ZEN, Grant n. 257660) and the Research Council of Norway (Norges Forskingsrådet) for the support. Silke Verbruggen gratefully acknowledges the financial support received from the Research Foundation-Flanders (FWO) in the frame of the strategic basic research project S009617N. Dolaana Khovalyg and Verena Barthelmes acknowledge support from the Swiss Federal Office of Energy (SFOE) as part of the project SI/501895-01. Marcel Schweiker was supported by a research grant (21055) from VILLUM FONDEN. Henrik N. Knudsen and Rune Korsholm Andersen would like to acknowledge funding by the Danish EUDP programme, project: 64 018-0558. The authors acknowledge TU Wien Bibliothek for financial support through its Open Access Funding Programme.

Acknowledgments: In the writing of this paper, the authors benefited from participation and related discussions in the IEA EBC Annex 79 activities.

Conflicts of Interest: The authors declare no conflict of interest.

\section{Appendix A}

In Scopus:

( TITLE ( "performance gap" OR "rebound" OR "prebound" OR "gap" AND NOT "knowledge gap" ) OR KEY ( "performance gap" OR "rebound" OR "prebound" ) AND TITLE-ABS-KEY ( building*) AND TITLE-ABS-KEY ( occupan* OR resident* OR user* OR uncertaint $^{*}$ ))

In Web of Science:

((TI=(("performance gap" OR "rebound" OR "prebound" OR "gap") NOT "knowledge gap*") OR (AK=("performance gap" OR "rebound" OR "prebound") ) AND (TI = (occupan* OR resident* OR user* OR uncertaint ${ }^{*}$ ) OR AB $=($ occupan* OR resident* OR user* OR uncertaint $\left.{ }^{*}\right)$ OR AK $=\left(\right.$ occupan $^{*}$ OR resident $*$ OR user* OR uncertaint $\left.\left.{ }^{*}\right)\right)$ AND (TI =(building*) $\mathrm{OR} \mathrm{AB}=\left(\right.$ building $\left.^{*}\right)$ OR AK=(building*)))

\section{Appendix B}

Appendix B entails a dynamic open-access review table that is available online at https: / / osf.io/dq9tj. This table includes further detailed information regarding all references with the "gold", "silver", and "bronze" labels (as defined in the paper). 


\section{References}

1. Mahdavi, A.; Berger, C. Predicting buildings' energy use: Is the occupant-centric "performance gap" research Program Ill-advised? Front. Energy Res. 2019, 7, 124. [CrossRef]

2. Mahdavi, A.; Taheri, M. Derivation and Representation of the Energetically Relevant Consequences of Occupants' Presence and Behaviour in Buildings. In Proceedings of the BauSim2018, Karlsruhe, Germany, 26-28 September 2018; Wagner, A., von Both, P., Eds.; pp. 216-222.

3. Scopus. Available online: https://www.scopus.com (accessed on 19 November 2020).

4. Web of Science. Available online: www.webofknowledge.com (accessed on 19 November 2020).

5. Lehmann, U.; Khoury, J.; Patel, M.K. Actual energy performance of student housing: Case Study, benchmarking and performance gap analysis. Energy Proc. 2017, 122, 163-168. [CrossRef]

6. Gill, Z.M.; Tierney, M.J.; Pegg, I.M.; Allan, N. Low-energy dwellings: The contribution of behaviours to actual performance. Build. Res. Inf. 2010, 38, 491-508. [CrossRef]

7. Jian, Y.; Li, Y.; Wei, S.; Zhang, Y.; Bai, Z. A case study on household electricity uses and their variations due to occupant behavior in Chinese apartments in Beijing. J. Asian Archit. Build. Eng. 2015, 14, 679-686. [CrossRef]

8. Burman, E.; Mumovic, D.; Kimpian, J. Towards measurement and verification of energy performance under the framework of the European directive for energy performance of buildings. Energy 2014, 77, 153-163. [CrossRef]

9. Guerra Santin, O.; Itard, L.; Visscher, H. The effect of occupancy and building characteristics on energy use for space and water heating in dutch residential stock. Energy Build. 2009, 41, 1223-1232. [CrossRef]

10. Delghust, M.; Laverge, J.; Janssens, A.; Cnockaert, E.; Davidson, T. The influence of energy performance levels on the heating demand in dwellings: Case-study analyses on neighbourhoods. Am. Soc. Heat. Refrig. Air Cond. Eng. 2013, 12, 9.

11. Dall'O', G.; Sarto, L.; Galante, A.; Pasetti, G. Comparison between predicted and actual energy performance for winter heating in high-performance residential buildings in the lombardy region (Italy). Energy Build. 2012, 47, 247-253. [CrossRef]

12. Housez, P.P.; Pont, U.; Mahdavi, A. A Comparison of projected and actual energy performance of buildings after thermal retrofit measures. J. Build. Phys. 2014, 38, 138-155. [CrossRef]

13. Yousefi, F.; Gholipour, Y.; Yan, W. A study of the impact of occupant behaviors on energy performance of building envelopes using occupants' data. Energy Build. 2017, 148, 182-198. [CrossRef]

14. Gupta, R.; Kapsali, M.; Howard, A. Evaluating the influence of building fabric, services and occupant related factors on the actual performance of low energy social housing dwellings in UK. Energy Build. 2018, 174, 548-562. [CrossRef]

15. Hahn, J.; Schumacher, P.; Lang, W.; Jensch, W. Performance gap and occupant behavior-review and analysis of high-efficiency residential buildings in Germany. In Proceedings of the 33rd International Conference on Efficiency, Cost, Optimization, Simulation and Environmental Impact of Energy Systems, ECOS 2020, Osaka, Japan, 29 June-3 July 2020; pp. $2010-2022$.

16. Carpino, C.; Loukou, E.; Heiselberg, P.; Arcuri, N. Energy Performance gap of a nearly zero energy building (NZEB) in Denmark: The influence of occupancy modelling. Build. Res. Inf. 2020, 1-23. [CrossRef]

17. Cuerda, E.; Guerra-Santin, O.; Sendra, J.J.; Neila González, F.J. Comparing the impact of presence patterns on energy demand in residential buildings using measured data and simulation models. Build. Simul. 2019, 12, 985-998. [CrossRef]

18. Hansen, A.R.; Gram-Hanssen, K.; Knudsen, H.N. How building design and technologies influence heat-related habits. Build. Res. Inf. 2018, 46, 83-98. [CrossRef]

19. Cayre, E.; Allibe, B.; Laurent, M.-H.; Osso, D. There are people in the house! How the results of purely technical analysis of residential energy consumption are misleading for energy policies. In Proceedings of the ECEEE 2011 Summer Study on Energy Efficiency: Energy Efficiency First: The Foundation of a Low-Carbon Society, Niagara Falls, NY, USA, 26-29 July 2011; pp. 1675-1683.

20. Aldossary, N.A. Domestic energy consumption patterns in a hot and humid climate: A multiple-case study analysis. Appl. Energy 2014, 13, 353-365. [CrossRef]

21. Vine, E.L.; Craig, P.P.; Cramer, J.C.; Dietz, T.M.; Hackett, B.M.; Kowalczyk, D.J.; Levine, M.D. The applicability of energy models to occupied houses: Summer electric use in davis. Energy 1982, 7, 909-925. [CrossRef]

22. Ali, Q.; Thaheem, M.J.; Ullah, F.; Sepasgozar, S.M.E. The Performance gap in energy-efficient office buildings: How the occupants can help? Energies 2020, 27, 1480. [CrossRef]

23. Masoso, O.T.; Grobler, L.J. The dark side of occupants' behaviour on building energy use. Energy Build. 2010, 42, 173-177. [CrossRef]

24. Brady, L. Assessment of energy consumption in existing buildings. Energy Build. 2017, 9, 142-150. [CrossRef]

25. Bros-Williamson, J.; Stinson, J.; Currie, J. Energy performance evaluation of a passive house built to scottish building standards. Int. J. Housing Sci. ITS Appl. 2015, 39, 11.

26. Delghust, M. Regulatory Energy calculations versus real energy use in high-performance houses. Build. Res. Inf. 2015, $43,17$. [CrossRef]

27. Galvin, R. Targeting 'behavers' rather than behaviours: A 'subject-oriented' approach for reducing space heating rebound effects in low energy dwellings. Energy Build. 2013, 12, 596-607. [CrossRef]

28. Grossmann, D.; Galvin, R.; Weiss, J.; Madlener, R.; Hirschl, B. A Methodology for estimating rebound effects in non-residential public service buildings: Case study of four buildings in Germany. Energy Build. 2016, 111, 455-467. [CrossRef] 
29. Guerra Santin, O. Occupant behaviour in energy efficient dwellings: Evidence of a rebound effect. J. Hous. Built Environ. 2013, 28, 311-327. [CrossRef]

30. Gupta, R.; Howard, A.; Kotopouleas, A. Meta-study of the energy performance gap in UK low energy housing. Buildings 2019, $12,1477-1487$.

31. Kane, T.; Firth, S.K.; Dimitriou, V.; Coleman, M.; Hassan, T. Exploring the Performance Gap in UK Homes: New Evidence from Smart Home and Smart Meter Data. In Proceedings of the BS2015: 14th Conference of International Building Performance Simulation Association, Hyderabad, India, 7-9 December 2015.

32. Littlewood, J.R.; Smallwood, I. Occupant Interaction with As-Designed Smart Heating: Impacts upon Energy Use \& Thermal Comfort. In KES-SEB 2018: Sustainability in Energy and Buildings 2018; Kaparaju, P., Howlett, R., Littlewood, J., Ekanyake, C., Vlacic, L., Eds.; Smart Innovation, Systems and Technologies; Springer: Cham, Germany, 2019; Volume 131. [CrossRef]

33. Majcen, D. Statistical model of the heating prediction gap in dutch dwellings: Relative importance of building, household and behavioural characteristics. Energy Build. 2015, 105, 17. [CrossRef]

34. Motamed, A.; Deschamps, L.; Scartezzini, J.-L. Eight-month experimental study of energy impact of integrated control of sun shading and lighting system based on HDR vision sensor. Energy Build. 2019, 203, 109443. [CrossRef]

35. Pegg, I.M.; Cripps, A.; Kolokotroni, M. Post-occupancy performance of five low-energy schools in the UK. ASHRAE Trans. 2007, 3,12 .

36. Salehi, M.M.; Terim Cavka, B.; Frisque, A.; Whitehead, D.; Bushe, W.K. A case study: The energy performance gap of the center for interactive research on sustainability at the University of British Columbia. J. Build. Eng. 2015, 4, 127-139. [CrossRef]

37. Moeller, S.; Weber, I.; Schröder, F.; Bauer, A.; Harter, H. Apartment related energy performance gap-how to address internal heat transfers in multi-apartment buildings. Energy Build. 2020, 215, 109887. [CrossRef]

38. Kim, Y.-S.; Heidarinejad, M.; Dahlhausen, M.; Srebric, J. Building energy model calibration with schedules derived from electricity use data. Appl. Energy 2017, 190, 997-1007. [CrossRef]

39. Mallory-Hill, S.; Gorgolewski, M. Mind the Gap: Studying actual versus predicted performance of green buildings in Canada. In Building Performance Evaluation; Preiser, W.F.E., Hardy, A.E., Schramm, U., Eds.; Springer International Publishing: Cham, Germany, 2018; pp. 261-274. [CrossRef]

40. Delghust, M.; Laverge, J.; Janssens, A.; Erck, C.V.; Taelman, C. The influence of user behaviour on energy use in old dwellings: Case-study analysis of a social housing neighbourhood. In Proceedings of the 5th International Building Physics Conference (IBPC 2012), Kyoto, Japan, 28-31 May 2012.

41. Azar, E.; Menassa, C.C. Optimizing the performance of energy-intensive commercial buildings: Occupancy-focused data collection and analysis approach. J. Comput. Civ. Eng. 2016, 30. [CrossRef]

42. van Dronkelaar, C.; Dowson, M.; Spataru, C.; Burman, E.; Mumovic, D. Quantifying the underlying causes of a discrepancy between predicted and measured energy use. Front. Mech. Eng. 2019, 5, 20. [CrossRef]

43. Bourdeau, M.; Guo, X.; Nefzaoui, E. Buildings energy consumption generation gap: A Post-occupancy assessment in a case study of three higher education buildings. Energy Build. 2018, 159, 600-611. [CrossRef]

44. Caceres, A.G.; Diaz, M. Usability of the EPC tools for the profitability calculation of a retrofitting in a residential building. Sustainability 2018, 10, 3159. [CrossRef]

45. Cuerda, E.; Guerra-Santin, O.; Sendra, J.J.; Neila, F.J. Understanding the performance gap in energy retrofitting: Measured input data for adjusting building simulation models. Energy Build. 2020, 209, 109688. [CrossRef]

46. Turner, C.; Frankel, M. Energy Performance of LEED for New Construction Buildings. Available online: https:/ / newbuildings. org/resource/energy-performance-leed-new-construction-buildings/ (accessed on 22 September 2020).

47. Herrando, M.; Cambra, D.; Navarro, M.; de la Cruz, L.; Millán, G.; Zabalza, I. Energy Performance certification of faculty buildings in Spain: The gap between estimated and real energy consumption. Energy Convers. Manag. 2016, 125, 141-153. [CrossRef]

48. Allard, I.; Olofsson, T.; Nair, G. Energy evaluation of residential buildings: Performance gap analysis incorporating uncertainties in the evaluation methods. Build. Simul. 2018, 11, 725-737. [CrossRef]

49. Calì, D.; Osterhage, T.; Streblow, R.; Müller, D. Energy Performance gap in refurbished German dwellings: Lesson learned from a field test. Energy Build. 2016, 127, 1146-1158. [CrossRef]

50. Jradi, M.; Arendt, K.; Sangogboye, F.C.; Mattera, C.G.; Markoska, E.; Kjærgaard, M.B.; Veje, C.T.; Jørgensen, B.N. ObepME: An online building energy performance monitoring and evaluation tool to reduce energy performance gaps. Energy Build. 2018, 166, 196-209. [CrossRef]

51. Menezes, A.C.; Cripps, A.; Bouchlaghem, D.; Buswell, R. Predicted vs. actual energy performance of non-domestic buildings: Using post-occupancy evaluation data to reduce the performance gap. Appl. Energy 2012, 97, 355-364. [CrossRef]

52. Gonzalez-Caceres, A.; Arvid Vik, T.; Granheim, P.; Fælth, J.P.; Berg, L. Improving the energy performance certificate recommendations' accuracy for residential building through simple measurements of key inputs. IOP Conf. Ser. Mater. Sci. Eng. 2019, 609, 032053. [CrossRef]

53. Gupta, R.; Kapsali, M. Evaluating the 'as-built' performance of an eco-housing development in the UK. Build. Service Eng. Res. Technol. 2016, 37, 220-242. [CrossRef]

54. Gupta, R.; Kapsali, M.; Gregg, M. Comparative building performance evaluation of a 'sustainable' community centre and a public library building. Build. Service Eng. Res. Technol. 2017, 38, 691-710. [CrossRef] 
55. Hamburg, A.; Kuusk, K.; Mikola, A.; Kalamees, T. Realisation of energy performance targets of an old apartment building renovated to NZEB. Energy 2020, 194, 116874. [CrossRef]

56. Heesen, F. Consumer behavior in energy-efficient homes: The limited merits of energy performance ratings as benchmarks. Energy Build. 2018, 9, 405-413. [CrossRef]

57. Jain, N.; Burman, E.; Robertson, C.; Stamp, S.; Shrubsole, C.; Aletta, F.; Barrett, E.; Oberman, T.; Kang, J.; Raynham, P.; et al. Building performance evaluation: Balancing energy and indoor environmental quality in a UK school building. Build. Service Eng. Res. Technol. 2020, 41, 343-360. [CrossRef]

58. Vetterli, N.; Sulzer, M.; Menti, U.-P. Energy monitoring of a low temperature heating and cooling district network. Energy Proc. 2017, 122, 62-67. [CrossRef]

59. Al-Mumin, A.; Khattab, O.; Sridhar, G. Occupants' behavior and activity patterns influencing the energy consumption in the kuwaiti residences. Energy Build. 2003, 35, 549-559. [CrossRef]

60. Van Someren, K.; Beaman, P.; Shao, L. Calculating the lighting performance gap in higher education classrooms. Int. J. Low-Carbon Technol. 2018, 13, 15-22. [CrossRef]

61. Imam, S.; Coley, D.A.; Walker, I. The building performance gap: Are modellers literate? Build. Service Eng. Res. Technol. 2017, 38, 351-375. [CrossRef]

62. Taylor, J.; Liu, Y.; Lin, B.; Burman, E.; Hong, S.-M.; Yu, J.; Wang, Z.; Mumovic, D.; Shrubsole, C.; Vermeer, D.; et al. Towards a framework to evaluate the 'total' performance of buildings. Build. Service Eng. Res. Technol. 2018, 39, 609-631. [CrossRef]

63. Azar, E.; Menassa, C.C. A Comprehensive framework to quantify energy savings potential from improved operations of commercial building stocks. Energy Policy 2014, 67, 459-472. [CrossRef]

64. Liu, P.; Lin, B.; Wu, X.; Zhou, H. Bridging energy performance gaps of green office buildings via more targeted operations management: A system dynamics approach. J. Environ. Manag. 2019, 238, 64-71. [CrossRef] [PubMed]

65. Li, C.; Hong, T.; Yan, D. An insight into actual energy use and its drivers in high-performance buildings. Appl. Energy 2014, 131, 394-410. [CrossRef]

66. De Wilde, P. The gap between predicted and measured energy performance of buildings: A framework for investigation. Autom. Constr. 2014, 41, 40-49. [CrossRef]

67. Al Amoodi, A.; Azar, E. Impact of human actions on building energy performance: A case study in the United Arab Emirates (UAE). Sustainability 2018, 10, 1404. [CrossRef]

68. Ahn, K.-U.; Kim, D.-W.; Park, C.-S.; de Wilde, P. Predictability of occupant presence and performance gap in building energy simulation. Appl. Energy 2017, 208, 1639-1652. [CrossRef]

69. Demanuele, C.; Tweddell, T.; Davies, M. Bridging the gap between predicted and actual energy performance in schools. In Proceedings of the World Renewable Energy Congress XI, Abu Dhabi, United Arab Emirates, 23-30 September 2010.

70. Coleman, S.; Robinson, J.B. Introducing the qualitative performance gap: Stories about a sustainable building. Build. Res. Inf. 2018, 46, 485-500. [CrossRef]

71. Majcen, D.; Itard, L.C.M.; Visscher, H. Theoretical vs. actual energy consumption of labelled dwellings in the Netherlands: Discrepancies and policy implications. Energy Policy 2013, 54, 125-136. [CrossRef]

72. Rouleau, J.; Gosselin, L.; Blanchet, P. Robustness of energy consumption and comfort in high-performance residential building with respect to occupant behavior. Energy 2019, 188, 115978. [CrossRef]

73. Sangalli, A.; Pagliano, L.; Causone, F.; Salvia, G.; Morello, E.; Erba, S. Behavioural change effects on energy use in public housing: A Case study. In Sustainability in Energy and Buildings; Littlewood, J., Howlett, R.J., Capozzoli, A., Jain, L.C., Eds.; Springer: Singapore, 2020; Volume 163, pp. 759-768. [CrossRef]

74. Sirombo, E.; Filippi, M.; Catalano, A.; Sica, A. Building monitoring system in a large social housing intervention in Northern Italy. Energy Proc. 2017, 140, 386-397. [CrossRef]

75. Kragh, J.; Rose, J.; Knudsen, H.N.; Jensen, O.M. Possible explanations for the gap between calculated and measured energy consumption of new houses. Energy Proc. 2017, 132, 69-74. [CrossRef]

76. Sunikka-Blank, M.; Galvin, R. Introducing the prebound effect: The gap between performance and actual energy consumption. Build. Res. Inf. 2012, 40, 260-273. [CrossRef]

77. Rouleau, J.; Gosselin, L.; Blanchet, P. Understanding energy consumption in high-performance social housing buildings: A case study from Canada. Energy 2018, 145, 677-690. [CrossRef]

78. Shi, X.; Si, B.; Zhao, J.; Tian, Z.; Wang, C.; Jin, X.; Zhou, X. Magnitude, causes, and solutions of the performance gap of buildings: A review. Sustainability 2019, 11, 937. [CrossRef]

79. Majcen, D.; Itard, L.; Visscher, H. Actual and theoretical gas consumption in dutch dwellings: What causes the differences? Energy Policy 2013, 61, 460-471. [CrossRef]

80. Van den Brom, P.; Meijer, A.; Visscher, H. Performance gaps in energy consumption: Household groups and building characteristics. Build. Res. Inf. 2018, 46, 54-70. [CrossRef]

81. Robinson, J.F.; Foxon, T.J.; Taylor, P.G. Performance gap analysis case study of a non-domestic building. Proc. Inst. Civil Eng. Eng. Sustain. 2015, 169, 31-38. [CrossRef]

82. Jones, R.V.; Fuertes, A.; de Wilde, P. The Gap Between Simulated and Measured Energy Performance: A Case Study Across Six Identical New-Build Flats in the UK. In Proceedings of the BS2015: 14th Conference of International Building Performance Simulation Association, Hyderabad, India, 7-9 December 2015. 
83. Loukou, E.; Heiselberg, P.K.; Jensen, R.L.; Johra, H. Energy performance evaluation of a nearly zero energy building and the reasons for the performance gap between expected and actual building operation. In IOP Conference Series: Earth and Environmental Science; IOP Publishing: Bristol, UK, 2019; Volume 352. [CrossRef]

84. Br, M. Estimating the influence of rebound effects on the energy-saving potential in building stocks. Energy Build. 2018, 13, 62-74.

85. Niu, S.; Pan, W.; Zhao, Y. A virtual reality integrated design approach to improving occupancy information integrity for closing the building energy performance gap. Sustain. Cities Soc. 2016, 27, 275-286. [CrossRef]

86. Sun, K.; Hong, T. A Framework for quantifying the impact of occupant behavior on energy savings of energy conservation measures. Energy Build. 2017, 146, 383-396. [CrossRef]

87. Galvin, R. Making the 'rebound effect' more useful for performance evaluation of thermal retrofits of existing homes: Defining the 'energy savings deficit' and the 'energy performance gap. ' Energy Build. 2014, 69, 515-524. [CrossRef]

88. Gouveia, J.P.; Palma, P. Harvesting big data from residential building energy performance certificates: Retrofitting and climate change mitigation insights at a regional scale. Environ. Res. Lett. 2019, 14. [CrossRef]

89. Mojic, I.; Lehmann, M.; van Velsen, S.; Haller, M. Immogap-analysis of the performance gap of apartment buildings. E3S Web Conf. 2019, 111, 04016. [CrossRef]

90. Hunter, G.; Hoyne, S.; Noonan, L. Evaluation of the space heating calculations within the irish dwelling energy assessment procedure using sensor measurements from residential homes. Energy Proc. 2017, 111, 181-194. [CrossRef]

91. Buso, T.; Fabi, V.; Andersen, R.K.; Corgnati, S.P. Occupant Behaviour and robustness of building design. Build. Environ. 2015, 94, 694-703. [CrossRef]

92. Niemierko, R.; Töppel, J.; Tränkler, T. A D-vine copula quantile regression approach for the prediction of residential heating Energy consumption based on historical data. Appl. Energy 2019, 233-234, 691-708. [CrossRef]

93. Bordass, B.; Cohen, R.; Standeven, M.; Leaman, A. Assessing building performance in use 3: Energy performance of the probe buildings. Build. Res. Inf. 2001, 29, 114-128. [CrossRef]

94. Berggren, B.; Wall, M. Two methods for normalisation of measured energy performance-testing of a net-zero energy building in Sweden. Buildings 2017, 7, 86. [CrossRef]

95. Cozza, S.; Chambers, J.; Geissler, A.; Wesselmann, K.; Gambato, C.; Branca, G.; Cadonau, G.; Arnold, L.; Patel, M. GAPxPLORE: Energy Performance Gap in Existing, New, and Renovated Buildings: Learning from Large-Scale Datasets; Office Fédéral de L'énergie: Berne, Switzerland, 2019.

96. Lee, S.; Jung, S.; Lee, J. Prediction model based on an artificial neural network for user-based building energy consumption in South Korea. Energies 2019, 12, 608. [CrossRef]

97. Bonte, M.; Thellier, F.; Lartigue, B. Impact of occupant's actions on energy building performance and thermal sensation. Energy Build. 2014, 76, 219-227. [CrossRef]

98. Energie en Duurzaamheid-Dossier. Available online: https://www.aedes.nl/dossiers/energie-en-duurzaamheid/energie-enduurzaamheid.html (accessed on 14 December 2020).

99. Levine, M.; Chen, S.; Yoshino, H.; Newhouse, K.; Hinge, A. Total Energy Use in Buildings Analysis and Evaluation Methods-Final Report of Annex 53: Definition of Terms-Separate Document Volume I; International Energy Agency: Paris, France, 2013.

100. American Society of Heating, Refrigerating and Air-Conditioning Engineers (ASHRAE). 2013 ASHRAE Handbook-Fundamentals (SI Edition); ASHRAE: Atlanta, GA, USA, 2013.

101. Hamburg, A.; Kalamees, T. How Well are energy performance objectives being achieved in renovated apartment buildings in Estonia? Energy Build. 2019, 199, 332-341. [CrossRef]

102. Khoury, J.; Hollmuller, P.; Lachal, B.M. Energy performance gap in building retrofit: Characterization and effect on the energy saving potential. In Proceedings of the 19. Status-Seminar «Forschen für den Bau im Kontext von Energie und Umwelt», Zurich, Switzerland, 8-9 September 2016.

103. SMHI. SMHI Energi Index. Available online: http://www.smhi.se/polopoly_fs/1.3499!/Menu/general/extGroup/ attachmentColHold/mainCol1/ file/Produktexempel\%20f\%C3\%B6rklaring\%20Energi\%20Index\%20151026.pdf (accessed on 14 December 2020).

104. Sonderegger, R.C. Movers and Stayers: The resident's contribution to variation across houses in energy consumption for space heating. Energy Build. 1978, 1, 313-324. [CrossRef]

105. Dott, R.; Haller, M.Y.; Ruschenburg, J.; Ochs, F.; Bony, J. A Technical Report of Subtask C Report C1 Part, B. In International Energy Agency (IEA) Solar Heating \& Cooling Programme (SHC) Task 44; International Energy Agency: Paris, France, 2013.

106. Johnston, D.; Siddall, M.; Ottinger, O.; Peper, S.; Feist, W. Are the energy savings of the passive house standard reliable? A review of the as-built thermal and space heating performance of passive house dwellings from 1990 to 2018. Energy Effic. 2020. [CrossRef]

107. Branco, G.; Lachal, B.; Gallinelli, P.; Weber, W. Predicted versus observed heat consumption of a low energy multifamily complex in Switzerland based on long-term experimental data. Energy Build. 2004, 36, 543-555. [CrossRef]

108. Lambie, E.; Senave, M.; Van de Vyver, I.; Saelens, D. Experimental analysis of indoor temperature of residential buildings as an input for building simulation tools. Energy Proc. 2017, 132, 123-128. [CrossRef]

109. Teli, D.; Dimitriou, T.; James, P.; Bahaj, A.; Ellison, L.; Waggott, A. Fuel poverty-induced 'prebound effect' in achieving the anticipated carbon savings from social housing retrofit. Build. Serv. Eng. Res. Technol. 2015, 37, 176-193. [CrossRef]

110. Liang, J.; Qiu, Y.; Hu, M. Mind the energy performance gap: Evidence from green commercial buildings. Resour. Conserv. Recycl. 2019, 141, 364-377. [CrossRef] 
111. Roetzel, A.; Tsangrassoulis, A.; Dietrich, U. Impact of building design and occupancy on office comfort and energy performance in different climates. Build. Environ. 2014, 71, 165-175. [CrossRef]

112. Clevenger, C.M.; Haymaker, J.R.; Jalili, M. Demonstrating the impact of the occupant on building performance. J. Comput. Civ. Eng. 2014, 28, 99-102. [CrossRef]

113. Zou, P.X.W.; Xu, X.; Sanjayan, J.; Wang, J. Review of 10 years research on building energy performance gap: Life-cycle and stakeholder perspectives. Energy Build. 2018, 178, 165-181. [CrossRef]

114. Kelly, S.; Shipworth, M.; Shipworth, D.; Gentry, M.; Wright, A.; Pollitt, M.; Crawford-Brown, D.; Lomas, K. Predicting the diversity of internal temperatures from the English residential sector using panel methods. Appl. Energy 2013, 102, 601-621. [CrossRef]

115. Haas, R.; Auer, H.; Biermayr, P. The impact of consumer behavior on residential energy demand for space heating. Energy Build. 1998, 11, 195-205. [CrossRef]

116. Gupta, R.; Dantsiou, D. Understanding the gap between 'as designed' and 'as built' performance of a new low carbon housing development in UK. In Sustainability in Energy and Buildings; Hakansson, A., Höjer, M., Howlett, R.J., Jain, L.C., Eds.; Springer: Berlin/Heidelberg, Germany, 2013; Volume 22, pp. 567-580. [CrossRef]

117. McElroy, D.J.; Rosenow, J. Policy implications for the performance gap of low-carbon building technologies. Build. Res. Inf. 2019, 47, 611-623. [CrossRef]

118. Mahdavi, A.; Tahmasebi, F. The deployment-dependence of occupancy-related models in building performance simulation. Energy Build. 2016, 117, 313-320. [CrossRef]

119. Mahdavi, A. In the matter of simulation and buildings: Some critical reflections. J. Build. Perform. Simul. 2020, 13, 26-33. [CrossRef] 